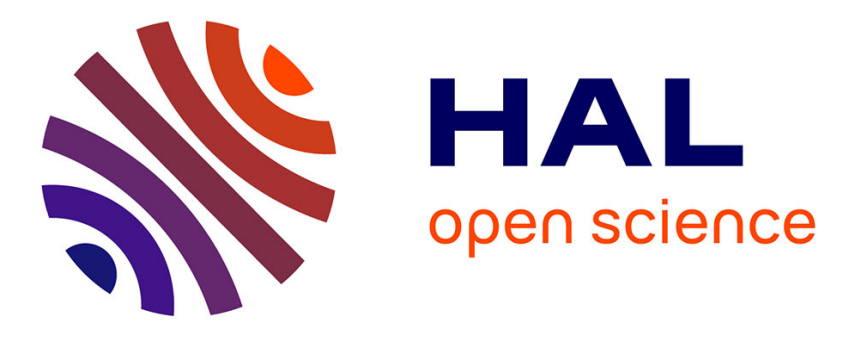

\title{
Near-field heat transfer between a nanoparticle and a rough surface
}

\author{
Svend-Age Biehs, Jean-Jacques Greffet
}

\section{To cite this version:}

Svend-Age Biehs, Jean-Jacques Greffet. Near-field heat transfer between a nanoparticle and a rough surface. Physical Review B: Condensed Matter and Materials Physics (1998-2015), 2010, 81 (24), pp.245414. 10.1103/PhysRevB.81.245414 . hal-00574373

\section{HAL Id: hal-00574373 \\ https://hal-iogs.archives-ouvertes.fr/hal-00574373}

Submitted on 7 Dec 2015

HAL is a multi-disciplinary open access archive for the deposit and dissemination of scientific research documents, whether they are published or not. The documents may come from teaching and research institutions in France or abroad, or from public or private research centers.
L'archive ouverte pluridisciplinaire $\mathbf{H A L}$, est destinée au dépôt et à la diffusion de documents scientifiques de niveau recherche, publiés ou non, émanant des établissements d'enseignement et de recherche français ou étrangers, des laboratoires publics ou privés. 


\title{
Near-field heat transfer between a nanoparticle and a rough surface
}

\author{
S.-A. Biehs and J.-J. Greffet \\ Laboratoire Charles Fabry, Institut d'Optique, CNRS, Université Paris-Sud, Campus Polytechnique, \\ RD128, 91127 Palaiseau Cedex, France
}

(Received 12 December 2009; published 10 June 2010)

\begin{abstract}
In this work we focus on the surface roughness correction to the near-field radiative heat transfer between a nanoparticle and a material with a rough surface utilizing a direct perturbation theory up to second order in the surface profile. We discuss the different distance regimes for the local density of states above the rough material and the heat flux analytically and numerically. We show that the heat transfer rate is larger than that corresponding to a flat surface at short distances. At larger distances it can become smaller due to surface polariton scattering by the rough surface. For distances much smaller than the correlation length of the surface profile, we show that the results converge to a proximity approximation, whereas in the opposite limit the rough surface can be replaced by an equivalent surface layer.
\end{abstract}

DOI: $10.1103 /$ PhysRevB.81.245414

PACS number(s): 44.40.+a, 68.35.Ct, 41.20.Jb, 05.40.-a

\section{INTRODUCTION}

Recently, the near-field radiative heat transfer has attracted a lot of theoretical and experimental attention. ${ }^{1-3}$ It was predicted theoretically ${ }^{4}$ and shown experimentally ${ }^{5-8}$ that the heat flux for distances much smaller than the thermal wavelength $\lambda_{\text {th }}=\hbar c /\left(k_{\mathrm{B}} T\right)$ can be much greater than that predicted by Planck's law, where $\hbar$ is Planck's constant, $k_{\mathrm{B}}$ is Boltzmann's constant, $c$ is the velocity of light in vacuum, and $T$ is the temperature. This unusual property might, for example, be exploited for thermophotovoltaics ${ }^{9-12}$ and nearfield scanning thermal microscopy. ${ }^{13,14}$

It is common knowledge that the radiative properties of a material depend not only on the material parameters but also on the surface roughness. ${ }^{15}$ While the effect on far-field properties has been widely studied, ${ }^{16}$ the impact of surface roughness on near-field heat transfer has not been considered so far. From the experimentalist's point of view, at least an estimate of the surface roughness correction is desirable since one is confronted with a certain degree of surface roughness in all experimental setups.

In this work we will study the near-field heat transfer between a nanoparticle considered to be an electric dipole and a material with a rough surface. Within this model the change in the local density of states (LDOS) above the material due to the surface roughness completely causes the change in the heat flux. Since, the near-field heat transfer between two semi-infinite bodies is also largely determined by the LDOS, we think that our results are not only restricted to the here discussed geometry but can also be utilized to get a rough estimate of the impact of surface roughness for configurations used in recent experimental setups. ${ }^{5-8}$ In addition, the LDOS also plays a fundamental role for other physical phenomena as it determines, for instance, the lifetime of atoms and molecules near a surface so that the here given results for the LDOS have a wider range of application.

The paper is organized as follows: In Sec. II we give a short description of the dipole model of near-field heat transfer. In Sec. III we introduce the perturbation result for the mean LDOS, the key quantity for understanding the roughness correction to the mean heat flux which is itself dis- cussed in Sec. IV. In Sec. V we derive approximations for the small and the large distance regime. Finally, in Sec. VI we study the roughness correction to the LDOS numerically.

\section{RADIATIVE HEAT TRANSFER}

We consider the situation depicted in Fig. 1. A nanoparticle with a polarizability $\alpha$ in local thermal equilibrium at temperature $T_{\mathrm{P}}$ is placed at $\mathbf{r}_{\mathrm{P}}$ near a dielectric half space with a given permittivity $\epsilon$. As discussed in Refs. 17 and 18 the multiple scattering between the nanoparticle and the surface can be neglected. We assume that this dielectric is in local thermal equilibrium at a temperature $T_{\mathrm{B}} \neq T_{\mathrm{P}}$. The interface separating the dielectric from the vacuum is described by the surface profile $S(\mathbf{x})-z=0$ with $\mathbf{x}=(x, y)$.

Now, as far as the radius $R$ of the nanoparticle is smaller than the thermal wavelength $\lambda_{\text {th }}$ and the distance $d$ between the dielectric body and the particle can be assumed to be large, i.e., $\lambda_{\text {th }} \gg R$ and $d \gg R$, the energy-transfer rate between the particle and the dielectric body due to radiation can be expressed within the dipole model as (see Refs. 2 and 3 and references therein)

$$
\left\langle P^{\mathrm{P} \leftrightarrow \mathrm{B}}\right\rangle=\int_{0}^{\infty} d \omega 2 \omega \alpha^{\prime \prime}(\omega)\left[\Theta\left(\omega, T_{\mathrm{P}}\right)-\Theta\left(\omega, T_{\mathrm{B}}\right)\right] D^{\mathrm{E}}\left(\omega, \mathbf{r}_{\mathrm{P}}\right)
$$

with

$$
\Theta(\omega, T)=\frac{\hbar \omega}{e^{\hbar \omega \beta}-1},
$$

where $\beta=\left(k_{\mathrm{B}} T\right)^{-1}$. Here, the spectral power absorbed by the nanoparticle $\mathrm{e}^{2,3,19}$ is given by the term $\alpha^{\prime \prime}(\omega) D^{\mathrm{E}}\left(\omega, \mathbf{r}_{\mathrm{P}}\right) \Theta\left(\omega, T_{\mathrm{B}}\right)$, i.e., it is proportional to the imaginary part of the polarizability $\alpha^{\prime \prime}$ of the particle and proportional to the electric energy density which is given by the product $D^{\mathrm{E}}\left(\omega, \mathbf{r}_{\mathrm{P}}\right) \Theta\left(\omega, T_{\mathrm{B}}\right)$ of the electric LDOS above the dielectric medium and the mean energy of a harmonic oscillator $\Theta(\omega, T)$. On the other hand, the power emitted by the particle and absorbed within the bulk medium is proportional 


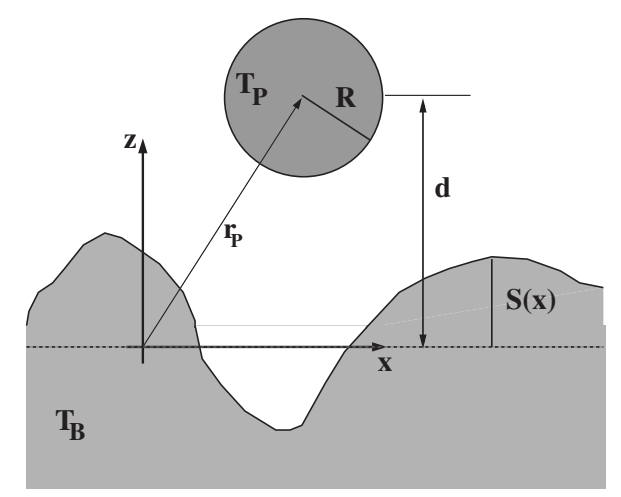

FIG. 1. Sketch of the configuration considered here.

to $\alpha^{\prime \prime}(\omega) D^{\mathrm{E}}\left(\omega, \mathbf{r}_{\mathrm{P}}\right) \Theta\left(\omega, T_{\mathrm{P}}\right)$. We point out that the above given formula has to be augmented by its magnetic counterpart when considering metallic materials as discussed in Ref. 20.

Within this work we will use the expression of the LDOS

$$
D^{\mathrm{E}}(\omega, \mathbf{r})=\frac{\omega}{\pi c^{2}} \operatorname{Im} \operatorname{Tr} \mathbb{G}^{\mathrm{E}}(\mathbf{r}, \mathbf{r}),
$$

as defined in Ref. 21. Using Eq. (3) in Eq. (1) corresponds to a situation where the bulk and its surrounding are at ambient temperature $T_{\mathrm{B}}$, whereas the nanoparticle is heated or cooled with respect to $T_{\mathrm{B}}$.

\section{LOCAL DENSITY OF STATES}

\section{A. Stochastic surface roughness}

In this work, we concentrate on the special case of a stochastic surface profile $S$ described as a stochastic Gaussian process with mean value and correlation function given by

$$
\begin{gathered}
\langle S(\mathbf{x})\rangle_{\mathrm{p}}=0, \\
\left\langle S(\mathbf{x}) S\left(\mathbf{x}^{\prime}\right)\right\rangle_{\mathrm{p}}=\delta^{2} W\left(\left|\mathbf{x}-\mathbf{x}^{\prime}\right|\right) .
\end{gathered}
$$

The brackets $\langle\circ\rangle_{\mathrm{p}}$ stand for the average over an ensemble of realizations of the surface profile $S(\mathbf{x}) ; \delta$ is the rms height of the surface profile. The correlation function $W\left(\left|\mathbf{x}-\mathbf{x}^{\prime}\right|\right)$ is here assumed to be given by a Gaussian

$$
W\left(\left|\mathbf{x}-\mathbf{x}^{\prime}\right|\right)=e^{-\left|\mathbf{x}-\mathbf{x}^{\prime}\right|^{2} / a^{2}},
$$

introducing the transverse correlation length $a$.

For the Fourier component $\tilde{S}(\boldsymbol{\kappa})$ of the surface-profile function one obtains

$$
\begin{gathered}
\langle\widetilde{S}(\boldsymbol{\kappa})\rangle_{\mathrm{p}}=0, \\
\left\langle\widetilde{S}(\boldsymbol{\kappa}) \tilde{S}\left(\boldsymbol{\kappa}^{\prime}\right)\right\rangle_{\mathrm{p}}=(2 \pi)^{2} \delta^{2} \delta\left(\boldsymbol{\kappa}+\boldsymbol{\kappa}^{\prime}\right) g(\boldsymbol{\kappa})
\end{gathered}
$$

with Dirac's delta function $\delta\left(\boldsymbol{\kappa}+\boldsymbol{\kappa}^{\prime}\right)$ and the surface roughness power spectrum

$$
g(\kappa)=\int d^{2} x W(|\mathbf{x}|) e^{-i \boldsymbol{\kappa} \cdot \mathbf{x}}=\pi a^{2} e^{-\kappa^{2} a^{2} / 4}
$$

\section{B. Perturbation expansion of the LDOS}

In order to determine the perturbation expansion of the LDOS in Eq. (3), we expand the Green's dyadic $\mathrm{G}^{\mathrm{E}}$ with respect to the surface profile up to second order. ${ }^{22}$ We follow Ref. 23, where one can find a procedure for determining the first-order Green's dyadic. The explicit second-order form is given in Appendix A. A detailed discussion of the validity of the perturbation theory is given in Ref. 23. In summary, the perturbation ansatz is valid as far as the surface height $\delta$ is the smallest length scale of the problem, i.e., $\delta$ $\ll \min \left\{z, a, \lambda_{\text {th }}\right\}$.

Inserting the Green's function up to second order given in Eq. (A1) into Eq. (3), we find for the LDOS up to second order after ensemble average,

$$
\begin{aligned}
\left\langle D^{\mathrm{E}^{(0)-(2)}}(\omega, \mathbf{r})\right\rangle_{\mathrm{p}} & \\
= & \frac{\omega}{\pi^{2} c^{2}}\left\{\int_{\kappa \leq k_{0}} d \kappa \frac{\kappa}{4 \gamma_{\mathrm{r}}}\left[1+h_{\mathrm{s}} \operatorname{Re}\left(\left\langle r_{\mathrm{s}}^{(0)-(2)}\right\rangle_{\mathrm{p}} e^{2 i \gamma_{\mathrm{r}} z}\right)\right]\right. \\
& \left.\quad+\int_{\kappa>k_{0}} d \kappa \frac{\kappa \mathrm{e}^{-2 \gamma z}}{4 \gamma}\left[h_{\mathrm{s}} \operatorname{Im}\left(\left\langle r_{\mathrm{s}}^{(0)-(2)}\right\rangle_{\mathrm{p}}\right)\right]+(s \leftrightarrow p)\right\},
\end{aligned}
$$

where $h_{\mathrm{s}}=1$ and $h_{\mathrm{p}}=\left(2 \kappa^{2}-k_{0}^{2}\right) / k_{0}^{2}$. The first term yields the propagating mode contribution, i.e., for $\kappa \leq k_{0}$ with $\gamma_{\mathrm{r}}$ $=\sqrt{k_{0}^{2}-\kappa^{2}}$ purely real, whereas the second term gives the contribution due to evanescent waves, i.e., $\kappa>k_{0}$ with $\gamma_{\mathrm{r}}$ $=i \gamma$ purely imaginary. The mean reflection coefficient up to second order $\left\langle r_{\mathrm{s} / \mathrm{p}}^{(0)-(2)}\right\rangle_{\mathrm{p}}$ can be written as a sum of the usual Fresnel coefficient $r_{\mathrm{s} / \mathrm{p}}$ and a surface roughness correction

$$
\left\langle r_{\mathrm{s} / \mathrm{p}}^{(0)-(2)}\right\rangle_{\mathrm{p}}=r_{\mathrm{s} / \mathrm{p}}-2 i \gamma_{\mathrm{r}}\left(D_{\mathrm{s} / \mathrm{p}}^{0}\right)^{2} M_{\mathrm{s} / \mathrm{p}},
$$

where

$$
D_{\mathrm{s}}^{0}=\frac{i}{\gamma_{\mathrm{r}}+\gamma_{\mathrm{t}}} \quad \text { and } \quad D_{\mathrm{p}}^{0}=\frac{i \epsilon}{\gamma_{\mathrm{r}} \epsilon+\gamma_{\mathrm{t}}}
$$

with $\gamma_{\mathrm{t}}=\sqrt{k_{0}^{2} \epsilon-\kappa^{2}}$. The so-called proper self-energy $M_{\mathrm{s} / \mathrm{p}}$ is defined in Appendix B. Furthermore, one can write the expression for the proper self-energy $M_{\mathrm{s} / \mathrm{p}}$ as a sum of two contributions $M_{\mathrm{s} / \mathrm{p}, 0}$ originating from terms due to a secondorder scattering process and $M_{\mathrm{s} / \mathrm{p}, 1}$ originating from terms due to two successive first-order scattering processes (see Fig. 2).

\section{RADIATIVE HEAT TRANSFER BETWEEN A NANOPARTICLE AND A ROUGH SURFACE}

With the above given relations we can study the radiative heat transfer between a spherical nanoparticle and a semiinfinite dielectric body with a rough surface formally given by Eq. (1) setting the nanoparticle at position $\mathbf{r}_{\mathrm{P}}=(0,0, d)$. For describing the absorptivity of the nanoparticle with radius $R$ we utilize the polarizability given as

$$
\alpha(\omega)=4 \pi R^{3} \frac{\epsilon(\omega)-1}{\epsilon(\omega)+2} .
$$

Here, we employ the material properties for SiC from Ref. 24 for numerical evaluation of Eq. (1) using Eq. (10) and 
(a)

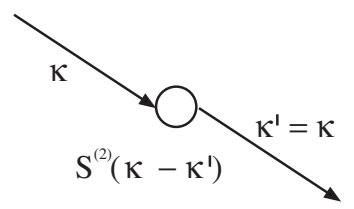

(b)

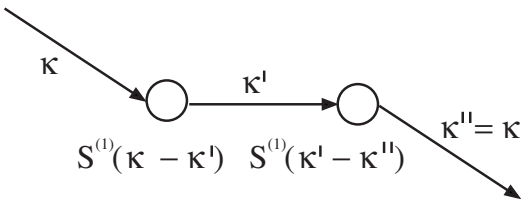

FIG. 2. Simple sketch of the scattering processes (a) due to $M_{\mathrm{s} / \mathrm{p}, 0}$ and (b) due to $M_{\mathrm{s} / \mathrm{p}, 1}$. After averaging the translational symmetry is restored so that $\boldsymbol{\kappa}$ is the same before and after the scattering with the rough surface. Mathematically, this property follows directly from the statistical properties of the Gaussian surface roughness, i.e., $\left\langle\widetilde{S}^{(2)}\left(\boldsymbol{\kappa}-\boldsymbol{\kappa}^{\prime}\right)\right\rangle_{\mathrm{p}} \propto \delta\left(\boldsymbol{\kappa}-\boldsymbol{\kappa}^{\prime}\right)$ and $\left\langle\widetilde{S}^{(1)}\left(\boldsymbol{\kappa}-\boldsymbol{\kappa}^{\prime}\right) \widetilde{S}^{(1)}\left(\boldsymbol{\kappa}^{\prime}\right.\right.$ $\left.\left.-\boldsymbol{\kappa}^{\prime \prime}\right)\right\rangle_{\mathrm{p}} \propto \delta\left(\boldsymbol{\kappa}-\boldsymbol{\kappa}^{\prime \prime}\right)$.

determine the surface roughness correction defined by

$$
\Delta P=100 \frac{\left\langle P^{(0)-(2)}\right\rangle_{\mathrm{p}}-P^{(0)}}{P^{(0)}} .
$$

We have checked that $P^{(0)}$ gives the same result as in Ref. 17 when choosing the same radius. (Note that $\Delta P$ itself does not depend on $R$ since $\left\langle P^{(0)-(2)}\right\rangle_{\mathrm{p}} \propto R^{3}$ and $P^{(0)} \propto R^{3}$.)

Let us first turn to the distance dependence of the heat flux. A plot of $\Delta P$ over the distance considering only evanescent modes, with $\delta=5 \mathrm{~nm}$ and $a=200 \mathrm{~nm}$ is shown in Fig. 3. The temperature of the dielectric is assumed to be $0 \mathrm{~K}$, whereas the temperature of the nanoparticle is set to $300 \mathrm{~K}$. From formula (1) it is clear that (apart from a sign for $P$ ) one gets the same result for $\Delta P$ when interchanging the temperatures. As will be shown later, the proximity approximation (PA)

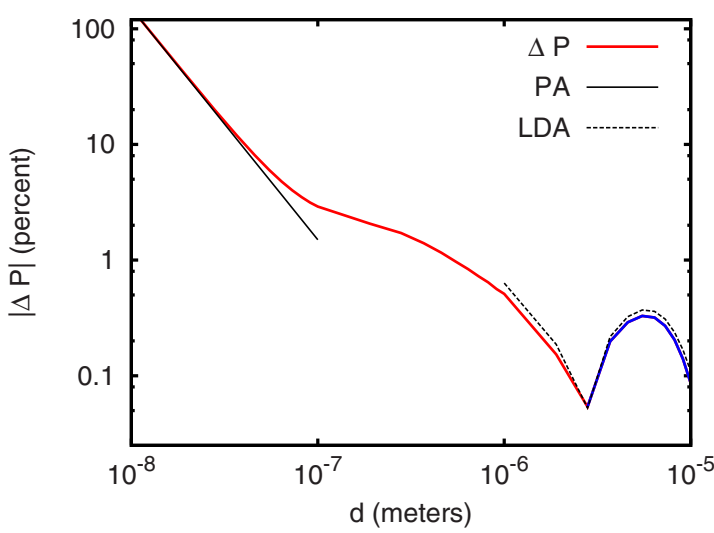

FIG. 3. (Color online) Plot of the modulus of $\Delta P$ for evanescent modes over the distance for $\mathrm{SiC}$ setting $T_{\mathrm{B}}=0 \mathrm{~K}$ and $T_{\mathrm{P}}=300 \mathrm{~K}$ for a rough surface with $\delta=5 \mathrm{~nm}$ and $a=200 \mathrm{~nm}$. The red part of the curve (for $d<2.8 \mu \mathrm{m}$ ) indicates positive and the blue one (for $d>2.8 \mu \mathrm{m})$ negative values. The PA and the LDA are included for comparison.

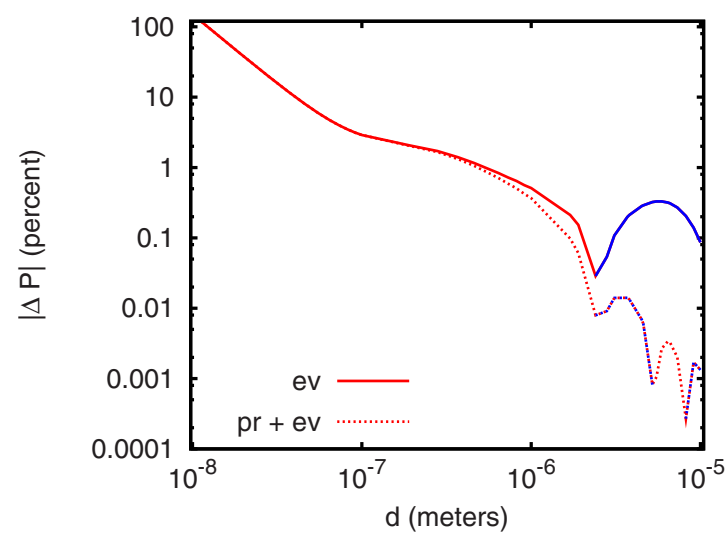

FIG. 4. (Color online) As Fig. 3 but for evanescent and propagating modes.

$$
\langle P\rangle_{\mathrm{p}}^{\mathrm{PA}}=P^{(0)}\left(1+6 \frac{\delta^{2}}{d^{2}}\right)
$$

can be derived in the small distance regime with $\delta \ll d \ll a$. Note that $\Delta P$ is always positive and independent from $T$ in that limit even if $\epsilon$ depends on $T$. In the opposite limit with $d \gg a$, we can also derive a large distance approximation (LDA) [see Eqs. (22) and (23)].

It can be seen, that $\Delta P$ converges to the approximations for large and small distances. For distances slightly greater than the small distance regime well described by the PA result the surface roughness correction becomes much greater than predicted by the PA. For distances between $1-10 \mu \mathrm{m}$ it can be seen that $\Delta P$ becomes negative. Now, in this distance regime already the propagating modes start to dominate the heat transfer. It turns out that for the propagating modes the surface roughness correction is very small compared to that of the evanescent modes. It follows that $\Delta P$ for evanescent and propagating modes becomes also very small in the distance regime $1-10 \mu \mathrm{m}$ as is illustrated in Fig. 4. It can also be seen that due to the competition of the roughness correction of the evanescent and propagating modes, the overall correction to the heat transfer $\Delta P$ becomes more oscillatory in that distance regime. Nevertheless, corrections on the order of $10 \%$ can arise for distances smaller than $100 \mathrm{~nm}$ but one has to keep in mind that the theory used here is only applicable for $d \gg \delta$ and $d \gg R$. Therefore, using $\delta=5 \mathrm{~nm}$ and $R=5 \mathrm{~nm}$ it can be estimated that the theory is only valid for distances $d$ greater than $\approx 50 \mathrm{~nm}$.

Since we have determined $P$ perturbatively with respect to the surface profile it is clear that to second order $\Delta P$ is proportional to $\delta^{2}$. On the other hand, the dependence of $\Delta P$ on the correlation length $a$ is not obvious. We find that $\Delta P$ is approximately proportional to $a^{-1}$ in the large distance regime (for the here used parameters) what might be seen in Fig. 5 considering only evanescent modes for $a=100,200$, and $500 \mathrm{~nm}$.

In order to understand the roughness correction to the heat flux, a deeper understanding of the roughness correction to the LDOS is required. Therefore, we will discuss the LDOS in more detail in the following. 


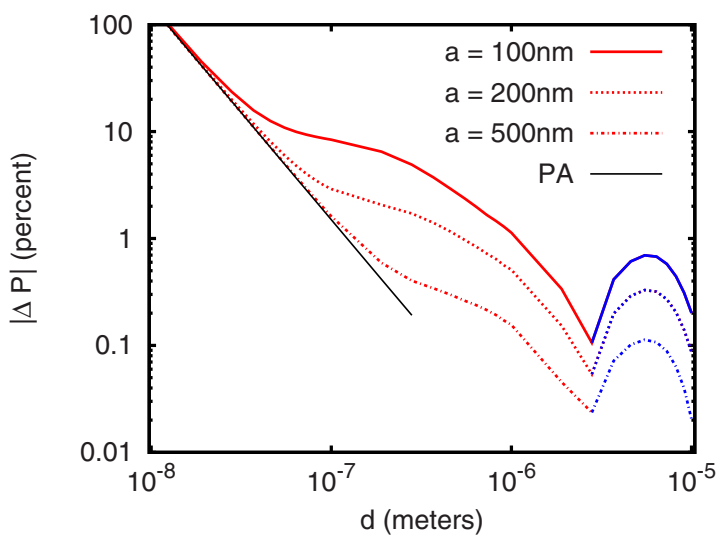

FIG. 5. (Color online) As Fig. 3 using $a=100,200$, and 500 nm.

\section{APPROXIMATIONS OF THE LDOS FOR SMALL AND LARGE DISTANCES}

The key quantities for the LDOS are the reflection coefficients in Eq. (11). Here we will derive some approximations of the proper self-energy $M_{\mathrm{s} / \mathrm{p}}$ first. From these expressions one can get the corresponding approximation for the LDOS by using Eqs. (10) and (11).

Before we derive the small and large distance approximation for the proper self-energy $M_{\mathrm{s} / \mathrm{p}}$ we first implement the following approximation: For frequencies relevant at room temperature, i.e., $\omega \approx 10^{14} \mathrm{~s}^{-1}$, we have $\left|k_{0} \sqrt{\epsilon} a / 2\right| \ll 1$, i.e., $a$ is much smaller than the skin depth $d_{\mathrm{s}}$, as far as the correlation length $a$ is small enough. Considering $\mathrm{SiC}$, this relation is well fulfilled for most frequencies within the range 3.7 $\times 10^{13}-2 \times 10^{14} \mathrm{~s}^{-1}$ for values of $a$ smaller than $500 \mathrm{~nm}$. Therefore, we concentrate here on the limit $a \ll d_{\text {s }}$ only (see Appendix C) while one can determine the opposite limit $a$ $\gg d_{\mathrm{s}}$ with a similar procedure. For the latter limit we just state the results in Appendix D.

\section{A. Small distance regime $(\delta \ll z \ll a)$}

In the limit of small distances $z$ it is seen from Eq. (10) that the main contribution comes from wave vectors $\kappa$ $\approx 1 / z$. In the regime $a \gg z(\kappa a \gg 1)$, the terms $M_{\mathrm{s} / \mathrm{p}, 1}$ become negligible compared to $M_{\mathrm{s} / \mathrm{p}, 0}$. This is due to the fact that for $\kappa a \gg 1$ the proper self-energy contributions $\left|M_{\mathrm{s} / \mathrm{p}, 1}\right|$ are proportional to $1 / \kappa$ or $\kappa$, respectively, because the Bessel functions are in this limit approximated by $\exp \left(\xi^{2} / 8\right) / \xi$. On the other hand, $\left|M_{\mathrm{s} / \mathrm{p}, 0}\right|$ is for large wave vectors proportional to $\kappa$ or $\kappa^{3}$ so that $\left|M_{\mathrm{s} / \mathrm{p}, 1}\right| /\left|M_{\mathrm{s} / \mathrm{p}, 0}\right| \propto 1 / \kappa^{2}$. This means that in the near-field regime the surface roughness correction solely stems from $M_{\mathrm{s} / \mathrm{p}, 0}$ in Eqs. (B10) and (B12), i.e., from that term which originates from one second-order scattering process (see also Fig. 2). Since the term $M_{\mathrm{s} / \mathrm{p}, 1}$ originating from terms due to two successive first-order scattering processes including processes involving the excitation of surface modes with $(\kappa, \omega)$ followed by scattering into another surface mode $\left(\kappa^{\prime}, \omega\right)$ as an intermediate state which is then scattered back into the initial state with $(\kappa, \omega)$ becomes negligible, we can formally conclude that these processes are irrelevant for $z \ll a$. This is very intuitive since the wave- length of the surface modes becomes much smaller than the correlation length. Within this limit the excited surface modes can follow the perturbed surface adiabatically without being scattered. Hence, for $z \ll a$ it suffices to consider $M_{\mathrm{s} / \mathrm{p}, 0}$ only. Inserting $M_{\mathrm{s} / \mathrm{p}, 0}$ into the mean reflection coefficient in Eq. (11) gives for the evanescent near-field regime with $\kappa$ $\gg k_{0}$

$$
\left\langle r_{\mathrm{s} / \mathrm{p}}^{(0)-(2)}\right\rangle_{\mathrm{p}} \approx \tilde{r}_{\mathrm{s} / \mathrm{p}}\left[1+2(\kappa \delta)^{2}\right],
$$

for $\kappa a \gg 1$ or $z \ll a$, utilizing the quasistatic approximations for the reflection coefficients

$$
\tilde{r}_{\mathrm{s}}=\frac{k_{0}^{2}}{4 \kappa^{2}}(\epsilon-1) \quad \text { and } \quad \tilde{r}_{\mathrm{p}}=\frac{\epsilon-1}{\epsilon+1} .
$$

From the above relations we can infer that the surface roughness correction for very small distances does not depend on $a$. The expressions in Eq. (16) can also be derived for $a$ $\gg d_{\mathrm{s}}$.

By inserting Eqs. (16) and (17) into the corresponding formula for the LDOS in the quasistatic limit

$$
\left.\left\langle D^{\mathrm{E}^{(0)-(2)}}\right\rangle_{\mathrm{p}} \approx \frac{\omega}{\pi^{2} c^{2}} \int_{0}^{\infty} d \kappa e^{-2 \kappa z} \frac{\kappa^{2}}{2 k_{0}^{2}} \operatorname{Im}\left(\left\langle r_{\mathrm{p}}^{(0)-(2)}\right)\right\rangle_{\mathrm{p}}\right),
$$

which follows from Eq. (10) when assuming $\kappa \gg k_{0}$, we find

$$
\left\langle D^{\mathrm{E}}(z)\right\rangle_{\mathrm{p}}^{\mathrm{PA}} \approx D^{\mathrm{E}^{(0)}}\left(1+6 \frac{\delta^{2}}{z^{2}}\right) .
$$

From this result the approximation for the radiative heat transfer in Eq. (15) follows easily.

Now, exactly the same result can be obtained with the so-called PA, which holds in the quasistatic limit for $z \ll a$ and $z \gg \delta$ which was, for example, used to determine the surface roughness contribution to the Casimir force ${ }^{25,26}$ and has recently been employed to the near-field radiative heat transfer. ${ }^{27}$ It amounts to replace the rough surface by a horizontal plane at a random height $z=S(\mathbf{x})$ followed by the ensemble average. Hence, the LDOS reads

$$
\begin{aligned}
\left\langle D^{\mathrm{E}}(z)\right\rangle_{\mathrm{p}} & \approx\left\langle D^{\mathrm{E}^{(0)}}(z-S(\mathbf{x}))\right\rangle_{\mathrm{p}} \\
& =D^{\mathrm{E}^{(0)}}(z)+\frac{1}{2} \frac{\partial^{2}}{\partial z^{2}} D^{\mathrm{E}^{(0)}} \delta^{2}+\mathcal{O}(4) .
\end{aligned}
$$

Utilizing the expression for the LDOS in the quasistatic $\operatorname{limit}^{21}$

$$
D^{\mathrm{E}^{(0)}} \approx \frac{1}{4} \frac{1}{\left(k_{0} z\right)^{3}} \frac{\omega^{2}}{\pi^{2} c^{3}} \frac{\epsilon^{\prime \prime}}{|\epsilon+1|^{2}},
$$

one retrieves the PA in Eq. (19) when considering terms up to second order only. Since the zeroth-order LDOS is proportional to $1 / z^{3}$, it is clear that the contributions for planes at distances smaller than $z$ will give a larger value than those at distances larger than $z$ so that after averaging the overall roughness correction is positive. Regarding only secondorder terms it is also clear that this correction is proportional to $\delta^{2}$. 

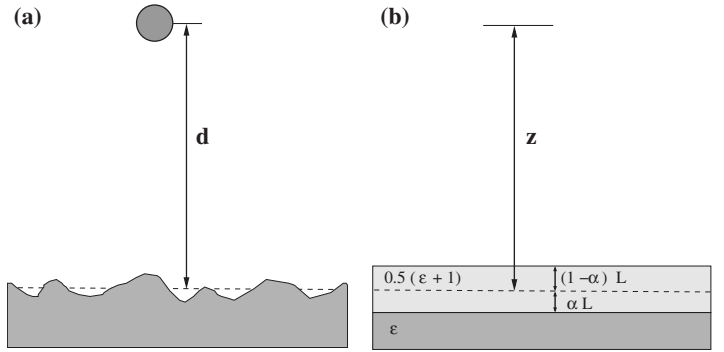

FIG. 6. (a) Sketch of the physical situation encountered in the large distance regime for the heat transfer and (b) the replacement of the rough surface by an equivalent layer for surface roughness to determine the LDOS in that regime.

\section{B. Large distance regime $\left(\lambda_{\mathrm{th}} \gg z \gg a\right.$ and $\left.a \ll d_{\mathrm{s}}\right)$}

When considering the evanescent contribution to the heat transfer in the large distance regime with $d \gg a$ one is facing a situation as sketched in Fig. 6(a). At room temperature $\lambda_{\text {th }} \approx 10 \mu \mathrm{m}$ so that the evanescent regime is restricted to $d \ll \lambda_{\text {th }}=10 \mu \mathrm{m}$. Therefore, this large distance regime is only applicable for surface profiles with a correlation length $a$ much smaller than $10 \mu \mathrm{m}$ and a rms $\delta \ll a$. The same is true for the LDOS at $z \gg a$. On the other hand, for very small temperatures of about $5 \mathrm{~K}$, the thermal wavelength is about $\lambda_{\text {th }} \approx 0.46 \mathrm{~mm}$. Hence, for low-temperature experiments as the measurement of spin-flip lifetimes in Refs. 28 and 29, this large distance regime can be applied to a much wider range of surface roughness parameters, distances and nanoparticle radii.

Considering the large distance limit $\kappa a \ll 1$ in Eq. (C1), we find for the lowest nonvanishing order

$$
M_{\mathrm{s}, 1} \approx-\left(k_{0} \delta\right)^{2}(\epsilon-1)^{2} \frac{\sqrt{\pi}}{2} \frac{1}{a} \frac{1}{\epsilon+1}
$$

and with Eq. (C4)

$$
M_{\mathrm{p}, 1} \approx \frac{\sqrt{\pi}}{2}\left(k_{0} \delta\right)^{2} \frac{(\epsilon-1)^{2}}{\epsilon(\epsilon+1)} \frac{1}{a}\left(1+\frac{\kappa^{2}}{k_{0}^{2}} \frac{2 \epsilon-1}{\epsilon}\right) .
$$

Obviously, $M_{\mathrm{s} / \mathrm{p}, 1}$ are proportional to $a^{-1}$. Therefore, for large distances for which $\left|M_{\mathrm{s} / \mathrm{p}, 1}\right| \gg\left|M_{\mathrm{s} / \mathrm{p}, 0}\right|$ is fulfilled, the secondorder correction to the LDOS will be inversely proportional to the correlation length if $a \ll d_{\mathrm{s}}$. The condition $\left|M_{\mathrm{s} / \mathrm{p}, 1}\right|$ $\gg\left|M_{\mathrm{s} / \mathrm{p}, 0}\right|$ is fulfilled for the $p$-polarized modes in the here given limit $\kappa a \ll 1$, whereas for the $s$-polarized modes, this is only true if $2 \gamma_{\mathrm{t}} a(\epsilon+1) \ll \sqrt{\pi}$. In addition, the approximate expressions in Eqs. (22) and (23) have the denominator $\epsilon+1$ indicating a strong contribution for surface resonances with $\epsilon^{\prime}=-1$ and $\epsilon^{\prime \prime} \approx 0$. Therefore, for frequencies near the surface resonance frequency and for distances $z \gg a$ the proper self-energy $M_{\mathrm{s} / \mathrm{p}}$ in the expressions for the reflection coefficient in Eq. (11) can be replaced by Eqs. (22) and (23) yielding the LDA.

As far as one considers correlation lengths $a_{-}$much smaller than the wavelength inside the medium $\lambda /|\sqrt{\epsilon}|$, one can apply the concept of homogenization ${ }^{30}$ by replacing the surface roughness by an equivalent surface layer with an effective permittivity. In the near-field regime, this condition is fulfilled if $\kappa a \ll 1$ or $a \ll z$, respectively. Following the approach of Rahman and Maradudin ${ }^{31,32}$ we replace the rough surface in the limit of large distances with $z \gg a$ and for $a$ $\ll d_{\text {s }}$ by a thin equivalent surface layer as depicted in Fig. 6 ranging from $z=-\alpha L$ to $(1-\alpha) L$ with $\alpha \in[0,1]$. The permittivity $\epsilon_{\mathrm{L}}$ of that layer is considered to be $0.5(\epsilon+1)$, i.e., the average of the permittivity of vacuum and the dielectric medium. The reflection coefficient for such a layered geometry with $\epsilon_{1}=\epsilon, \epsilon_{2}=\epsilon_{\mathrm{L}}$, and $\epsilon_{3}=1$ is in the quasistatic limit $\left(\kappa \gg k_{0}\right)$

$$
r_{\mathrm{s} / \mathrm{p}}^{\mathrm{L}} \approx e^{2 \kappa(1-\alpha) L} \frac{r_{\mathrm{s} / \mathrm{p}}^{32}+r_{\mathrm{s} / \mathrm{p}}^{21} e^{-2 \kappa L}}{1+r_{\mathrm{s} / \mathrm{p}}^{32} r_{\mathrm{s} / \mathrm{p}}^{21} e^{-2 \kappa L}} .
$$

Considering first $p$-polarized modes we insert the electrostatic expressions for the Fresnel coefficients

$$
r_{\mathrm{p}}^{32} \approx \frac{\epsilon_{\mathrm{L}}-1}{\epsilon_{\mathrm{L}}+1} \quad \text { and } \quad r_{\mathrm{p}}^{21} \approx \frac{\epsilon-\epsilon_{\mathrm{L}}}{\epsilon+\epsilon_{\mathrm{L}}}
$$

and expand the reflection coefficient for the layered system with respect to the thickness $L$, which is thought to be small, yielding

$$
r_{\mathrm{p}}^{\mathrm{L}} \approx \frac{\epsilon-1}{\epsilon+1}+\kappa L \frac{(\epsilon-1)}{(\epsilon+1)^{3}}\left[-2 \alpha(1+\epsilon)^{2}+2 \epsilon^{2}+\epsilon+1\right] .
$$

Now, we want to compare $\Delta r_{\mathrm{p}}^{\mathrm{L}}=r_{\mathrm{p}}^{\mathrm{L}}-r_{\mathrm{p}}$ with the result of the LDA. Inserting Eq. (23) into the expression for the mean reflection coefficients in Eq. (11) gives for the quasistatic limit $\left(\kappa \gg k_{0}\right)$

$$
\Delta\left\langle r_{\mathrm{p}}^{(0)-(2)}\right\rangle_{\mathrm{p}}=\left\langle r_{\mathrm{p}}^{(0)-(2)}\right\rangle_{\mathrm{p}}-r_{\mathrm{p}} \approx \sqrt{\pi} \frac{\kappa \delta^{2}}{a} \frac{(\epsilon-1)^{2}}{(\epsilon+1)^{3}}(2 \epsilon-1) .
$$

In order to get a relation between $L$ and $\alpha$, we compare the leading term in $\epsilon$ for $\delta r_{\mathrm{p}}^{\mathrm{L}}$ of the layered geometry with the surface roughness correction. This gives

$$
(1-\alpha) L=\frac{\sqrt{\pi} \delta^{2}}{a} .
$$

Obviously, the choice of the parameters $\alpha$ and $L$ is ambiguous. This ambiguity is resolved in Ref. 31 when further considering the transmitted field component for the equivalent layer and the corresponding perturbative result. From this, Rahman and Maradudin find $L=3 \sqrt{\pi} \delta^{2} / a$. Using this result in Eq. (28) gives $\alpha=2 / 3$ as was also found by Rahman and Maradudin in Ref. 31. Hence, the surface roughness can be mimicked by a small layer with a thickness of order $\delta^{2} / a$ which is slightly shifted into the vacuum region with respect to $z=0$ so that the effective distance from the surface becomes $z-L / 3$. It follows that for distances $z \gg a$ the evanescent part of the LDOS and of the heat transfer, which have a monotonous decay with $z$, will be slightly bigger than that of a flat surface and proportional to $a^{-1}$.

The same considerations can be made for the $s$-polarized modes yielding the results 

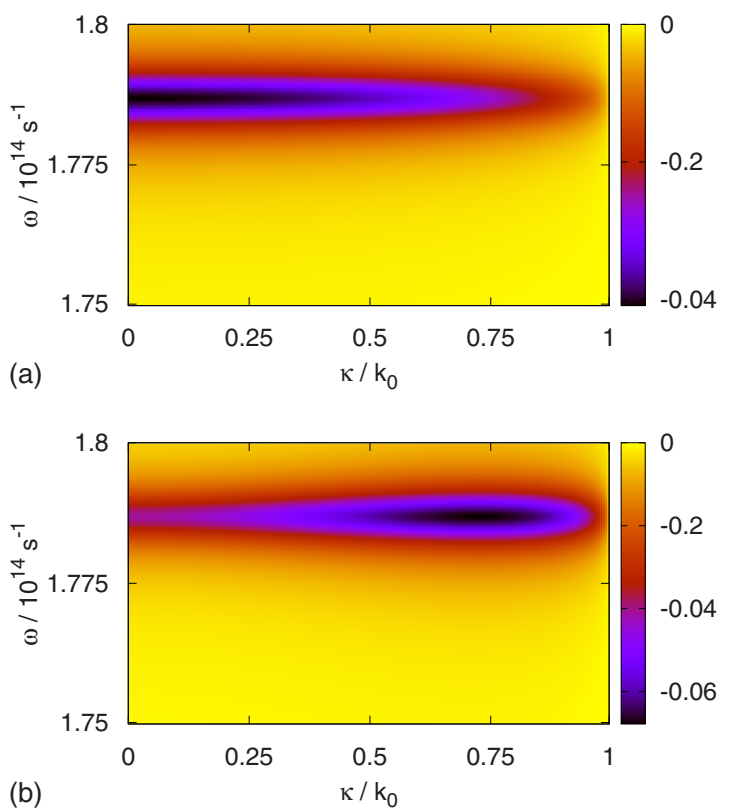

FIG. 7. (Color online) Plot of $\Delta R_{\mathrm{s} / \mathrm{p}}$ as defined in Eq. (32) for (a) $s$ - and (b) $p$-polarized modes using $\delta=5 \mathrm{~nm}$ and $a=200 \mathrm{~nm}$.

$$
\Delta r_{\mathrm{s}}^{\mathrm{L}} \approx \frac{k_{0}^{2} L}{4 \kappa}(1-\epsilon)(1-2 \alpha)
$$

in the quasistatic regime, which can be compared to the corresponding correction to the mean reflection coefficient [by inserting Eq. (22) into Eq. (11)]

$$
\Delta\left\langle r_{\mathrm{s}}^{(0)-(2)}\right\rangle_{\mathrm{p}} \approx-\left(k_{0} \delta\right)^{2} \sqrt{\pi} \frac{1}{\kappa a} \frac{1}{4} \frac{(\epsilon-1)^{2}}{(\epsilon+1)} .
$$

Comparing the leading-order term of $\delta r_{\mathrm{s}}^{\mathrm{L}}$ and $\delta\left\langle r_{\mathrm{s}}^{(0)-(2)}\right\rangle_{\mathrm{p}}$ in $\epsilon$ gives

$$
(1-2 \alpha) L=\frac{\sqrt{\pi} \delta^{2}}{a} .
$$

Again, the choice of $\alpha$ and $L$ is ambiguous. Using again the results for $L$ from Ref. 31 gives $\alpha=1 / 3$. Therefore, we get a similar result as for the p-polarized modes.

As it might be shown in the next section, in the large distance regime, the roughness plays a significant role only at the surface polariton resonance, i.e., for $\omega_{\mathrm{s}}$ such that $\operatorname{Re}\left[\epsilon\left(\omega_{\mathrm{s}}\right)\right]+1 \approx 0$ and $\operatorname{Im}\left[\epsilon\left(\omega_{\mathrm{s}}\right)\right] \approx 0$. This is clear when noting the resonant denominator $1 /(\epsilon+1)^{3}$ in Eq. (27). Hence, we expect a significant modification of the LDOS and therefore also of the lifetime of an emitter whose frequency is close to $\omega_{\mathrm{s}}$. On the other hand, for the near-field heat transfer one has to sum up all contributions over the spectrum close to the thermal frequency so that one can in general expect that the correction is in this case small.

Finally, we note that, although the physics is different, the effect of roughness on heat transfer is the same for evanescent and propagating waves. The roughness can be modeled by an effective layer with intermediate optical properties. In both cases, it results in a larger transmission.

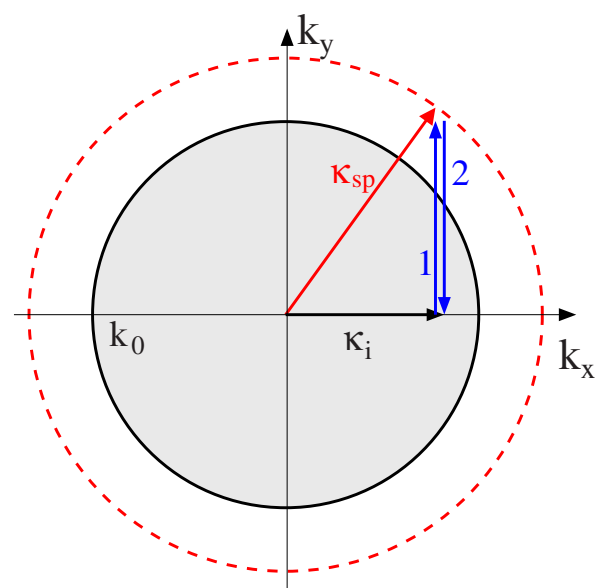

FIG. 8. (Color online) Illustration of the excitation of surface polaritons by $s$-polarized light in the $\boldsymbol{\kappa}$ plane (dashed line $=$ dispersion relation of the surface phonon polariton, gray circle =propagating waves with $\left.\kappa<k_{0}\right)$ : an $s$-polarized wave with $\boldsymbol{\kappa}_{i}$ $=\left(k_{i}, 0\right)$ and an electric field in $y$ direction. Due to a first scattering process labeled as 1 with the rough surface the surface power spectrum provides the necessary extra momentum $\left(\boldsymbol{\kappa}_{i}-\boldsymbol{\kappa}_{\text {sp }}\right)$ to match the phase with the surface polariton with wave vector $\boldsymbol{\kappa}_{\mathrm{sp}}$. The incoming $s$-polarized wave has an electric field component in the direction of $\boldsymbol{\kappa}_{\text {sp }}$ so that it can excite the surface polariton. Due to the second scattering process 2 the surface power spectrum again provides the necessary extra momentum $-\left(\boldsymbol{\kappa}_{i}-\boldsymbol{\kappa}_{\mathrm{sp}}\right)$ resulting in a scattered $s$-polarized wave with $\boldsymbol{\kappa}=\boldsymbol{\kappa}_{i}$.

\section{NUMERICAL RESULTS FOR THE LDOS}

\section{A. Propagating modes}

First, we discuss some numerical results for the propagating modes using the material parameters for SiC from Ref. 24. In Fig. 7, we show a plot of the deviation of reflectivity from Eq. (11) defined as

$$
\Delta R_{\mathrm{s} / \mathrm{p}}=100 \frac{\left|\left\langle r_{\mathrm{s} / \mathrm{p}}^{(0)-(2)}\right\rangle_{\mathrm{p}}\right|^{2}-\left|r_{\mathrm{s} / \mathrm{p}}\right|^{2}}{\left|r_{\mathrm{s} / \mathrm{p}}\right|^{2}}
$$

for $s$ and $p$ polarization for $\kappa \leq k_{0}$ choosing a rms of $\delta$ $=5 \mathrm{~nm}$ and a correlation length of $a=200 \mathrm{~nm}$. Furthermore, the plot is restricted to frequencies around the surface phonon polariton resonance $\omega_{\mathrm{s}}=1.787 \times 10^{14} \mathrm{~s}^{-1}$ within the reststrahlen band, i.e., $\omega_{\mathrm{t}}<\omega<\omega_{1}$, where $\omega_{1}=1.827 \times 10^{14} \mathrm{~s}^{-1}$ and $\omega_{\mathrm{t}}=1.495 \times 10^{14} \mathrm{~s}^{-1}$ are the frequencies of the LO and $\mathrm{TO}$ phonons in $\mathrm{SiC}$, respectively. It can be seen that the correction is small and negative in this frequency range. For frequencies around the surface phonon frequency one finds a relatively large negative correction (but still smaller than $1 \%$ ) for both polarizations. Hence, the roughness correction to the LDOS for propagating modes will be very small as well.

The surface roughness allows for coupling of incident propagating waves with surface polaritons. ${ }^{33-35}$ Hence, for $(\omega, \kappa)$ for which the conditions for coupling of surface polaritons with propagating modes are met, the reflectivity will decrease, i.e., $\Delta R_{\mathrm{s} / \mathrm{p}}$ is negative since only a small fraction of 

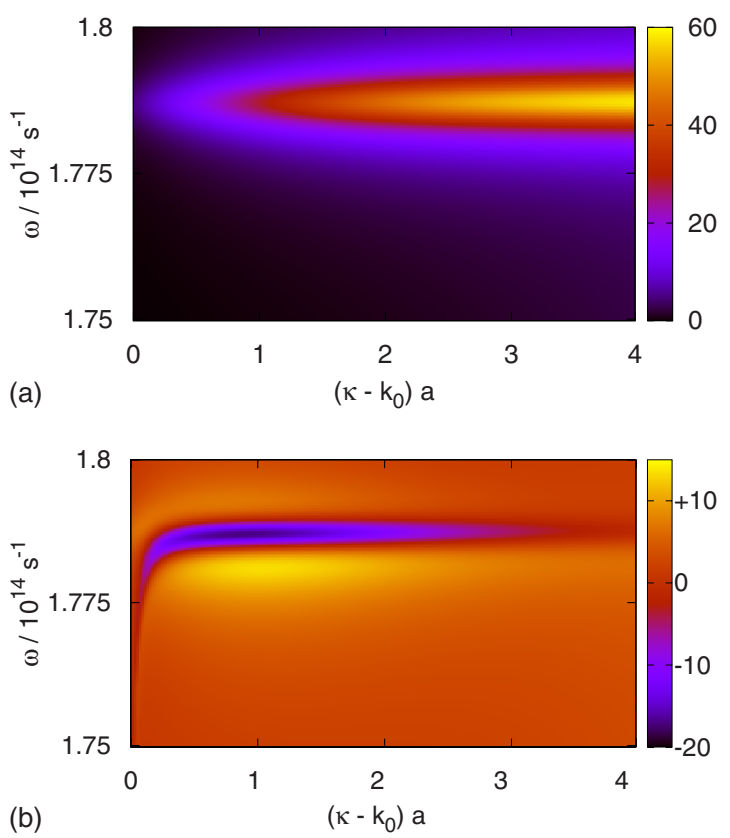

FIG. 9. (Color online) Plot of $\operatorname{Im}\left(r_{\mathrm{s} / \mathrm{p}}\right)$ as defined in Eq. (33) for (a) $s$ - and (b) $p$-polarized modes using $\delta=5 \mathrm{~nm}$ and $a=200 \mathrm{~nm}$.

the excited surface mode will be reradiated. This surface wave mediated decrease in the reflectivity can be seen for both $s$ and $p$ polarizations.

The possibility of exciting surface polaritons with $s$-polarized light needs some explanation since after taking the ensemble average, the rough surface has the same symmetries as a flat surface for which surface polaritons can be excited with $p$-polarized light only. On the other hand, for surfaces with a grating which breaks the translational and rotational symmetry, it is known that ${ }^{36,37} s$-polarized waves can excite surface polaritons. In this case, the grating provides the extra momentum for matching the phase of the incoming light with that of the surface polariton. Additionally, the incoming wave must have an electric field vector component parallel to the surface phonon polariton wave vector. When these two conditions are met, ${ }^{36,37}$ an $s$-polarized wave can excite surface polaritons. Now, for a rough surface the translational and rotational symmetries are also broken so that an $s$-polarized wave should be able to excite surface polaritons. An example of such a scattering process is illustrated and discussed in Fig. 8.

\section{B. Evanescent modes}

Now, we turn to the evanescent modes. According to Eq. (10), we are interested in $\operatorname{Im}(r)$. In order to discuss the change in $\operatorname{Im}(r)$ we define

$$
\Delta \operatorname{Im}\left(r_{\mathrm{s} / \mathrm{p}}\right)=100 \frac{\operatorname{Im}\left(\left\langle r_{\mathrm{s} / \mathrm{p}}^{(0)-(2)}\right\rangle_{\mathrm{p}}\right)-\operatorname{Im}\left(r_{\mathrm{s} / \mathrm{p}}\right)}{\operatorname{Im}\left(r_{\mathrm{s} / \mathrm{p}}\right)} .
$$

In Fig. 9 we show a plot of this quantity for $s$ - and $p$-polarized modes using again the parameters $\delta=5 \mathrm{~nm}$ and $a=200 \mathrm{~nm}$. We show here only the results for $\kappa a \leq 4$, i.e., in that region where the mean reflection coefficient is domi-

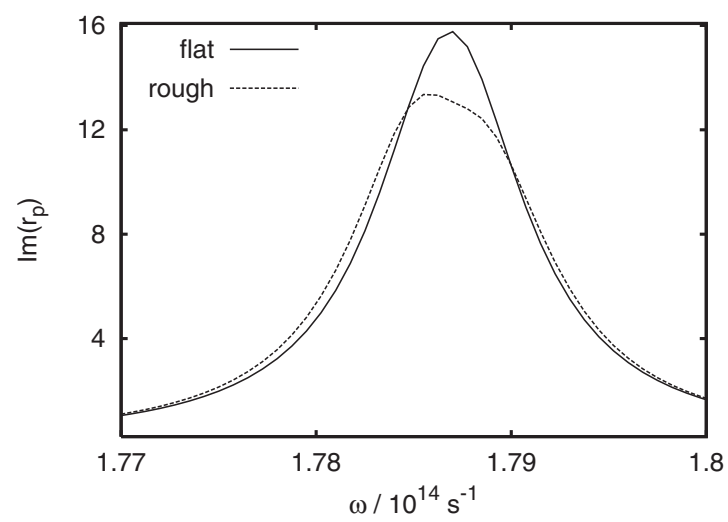

FIG. 10. Plot of $\operatorname{Im}\left(r_{\mathrm{p}}\right)$ and $\operatorname{Im}\left(\left\langle r_{\mathrm{p}}^{(0)-(2)}\right\rangle_{\mathrm{p}}\right)$ for $\kappa a=1$ using $\delta$ $=5 \mathrm{~nm}$ and $a=200 \mathrm{~nm}$.

nated by $M_{\mathrm{s} / \mathrm{p}, 1}$. For $s$-polarized modes one can see that $\operatorname{Im}\left(r_{\mathrm{s}}\right)$ is increased up to $60 \%$ for values of $(\omega, \kappa)$ coinciding with the dispersion relation of surface phonons. The underlying mechanism is the same as for the propagating modes depicted in Fig. 8 but with the difference that $\boldsymbol{\kappa}_{i}$ is greater than $k_{0}$.

On the other hand, for $p$-polarized modes $\operatorname{Im}\left(r_{\mathrm{p}}\right)$ is decreased by about $20 \%$ for values of $(\omega, \kappa)$ coinciding with the dispersion relation of the surface phonons. Around $\kappa a$ $\approx 1$ one also finds a large increase of about $15 \%$ for frequencies slightly below and above the surface phonon frequency. This can be easily understood by looking at Fig. 10 showing a plot of $\operatorname{Im}\left(r_{\mathrm{p}}\right)$ and $\operatorname{Im}\left(\left\langle r_{\mathrm{p}}^{(0)-(2)}\right\rangle\right)$ for $\kappa a=1$ and for frequencies near the surface phonon frequency. As can be observed, the dispersion relation is broadened due to roughness induced scattering of the surface phonons into other surface phonon states. For a slightly rougher surface with $\delta$ $=10 \mathrm{~nm}$ the broadening becomes a splitting of the surface polariton dispersion, ${ }^{32,38}$ which can be easily understood from the fact that the rough surface acts as a thin layer as discussed above. It follows, that the quantity $\Delta \operatorname{Im}\left(r_{\mathrm{s} / \mathrm{p}}\right)$ has negative values for frequencies around the surface phonon frequency and positive values slightly below and above that surface phonon frequency as can be seen in Fig. 9. We remark that for $\delta=10 \mathrm{~nm}$ the direct perturbation theory, while qualitatively correct, starts to give quantitatively wrong results for frequencies near the surface resonance frequency. ${ }^{39}$

\section{Spectrum of the LDOS}

From the above discussion it is clear that the roughness correction of the LDOS defined as

$$
\Delta D^{\mathrm{E}}=100 \frac{\left\langle D^{\mathrm{E}^{(0)-(2)}}\right\rangle_{\mathrm{P}}-D^{\mathrm{E}^{(0)}}}{D^{\mathrm{E}^{(0)}}}
$$

will have positive and negative contributions for different frequencies and distances. To make this point clear, we show some plots of $\Delta D^{\mathrm{E}}$ and $D^{\mathrm{E}}=\left\langle D^{\mathrm{E}^{(0)-(2)}}\right\rangle_{\mathrm{P}}$ for frequencies ranging from $10^{13}$ to $2.5 \times 10^{14} \mathrm{~s}^{-1}$.

In Fig. 11 we plotted $D^{\mathrm{E}}$ and $\Delta D^{\mathrm{E}}$ for propagating and evanescent modes and for propagating modes only for a distance of $z=5 \mu \mathrm{m}$. It is seen that $D^{\mathrm{E}}$ is dominated by the 


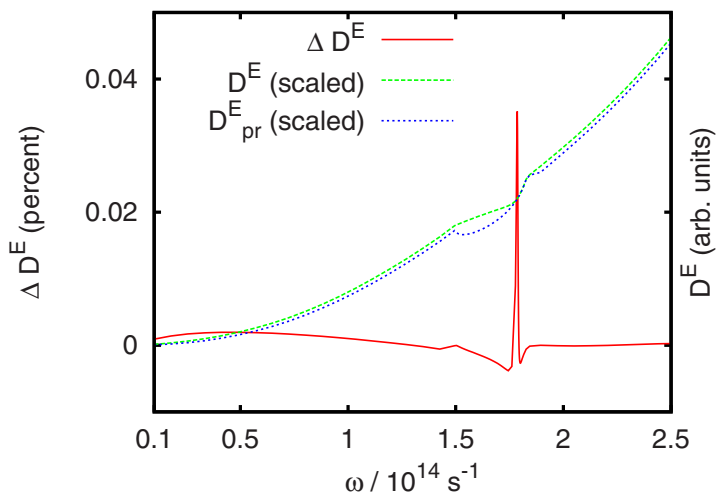

FIG. 11. (Color online) Plot of $D^{\mathrm{E}}$ and $\Delta D^{\mathrm{E}}$ as defined in Eqs. (10) and (34) for propagating and evanescent modes (dashed line) and for propagating modes only (dotted line) using $\delta=5 \mathrm{~nm}$ and $a=200 \mathrm{~nm}$ and distance $z=5 \mu \mathrm{m}$.

propagating modes and shows a small dip in the reststrahlen band of $\mathrm{SiC}$, where the contribution of the evanescent modes is already relatively large. The surface roughness correction is in this case extremely small and originates in equal parts from the correction to the evanescent and propagating modes.

For very small distances the LDOS is solely dominated by the evanescent contribution so that in this case the spectrum is quite different from that of the propagating part. ${ }^{21,24}$ In Fig. 12 we show a plot of $D^{\mathrm{E}}$ for $z=500 \mathrm{~nm}$, i.e., in the evanescent regime, leaving the other parameters unchanged. As can be seen, the spectrum of $D^{\mathrm{E}}$ has a resonance due to surface phonons. The curve of $\Delta D^{\mathrm{E}}$ shows a negative deviation of about $12 \%$ at the surface phonon resonance and a positive deviation of about $6 \%$ slightly below and above that resonance as can be seen in the inset. This behavior is due to the scattering of surface phonons discussed in the previous section resulting in a broadened surface phonon dispersion.

\section{Distance dependence of the LDOS}

Let us now focus on the distance dependence of the LDOS. For rough surfaces it can be expected that the rough-

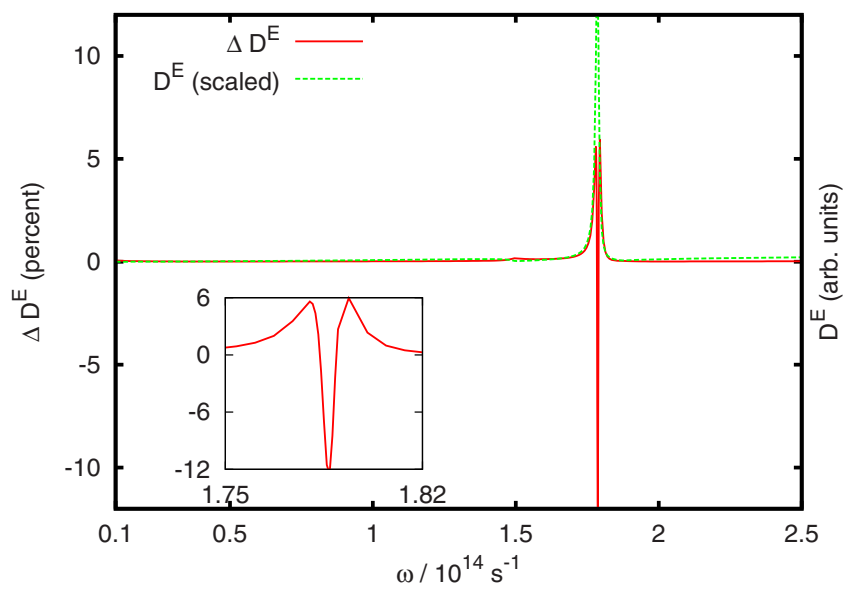

FIG. 12. (Color online) Plot of $D^{\mathrm{E}}$ and $\Delta D^{\mathrm{E}}$ as defined in Eq. (34) for $z=500 \mathrm{~nm}$ using the same roughness parameters as in Fig. 11.

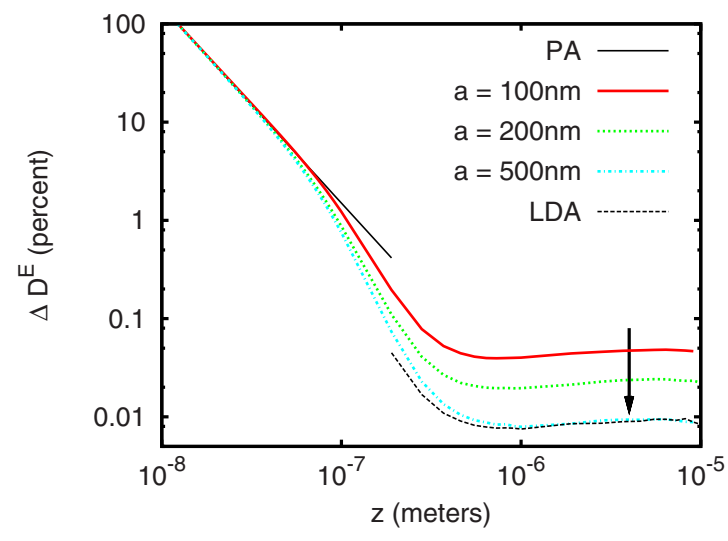

FIG. 13. (Color online) Plot of $\Delta D^{\mathrm{E}}$ over the distance for $\mathrm{SiC}$ using the frequency $\omega=10^{14} \mathrm{~s}^{-1}$ for a rough surface with $\delta$ $=5 \mathrm{~nm}$ for correlation length $a=100,200$, and $500 \mathrm{~nm}$. The thin solid line represents the PA result, whereas the thin dashed-dotted line is the LDA.

ness correction of the LDOS $\Delta D^{\mathrm{E}}$ defined in Eq. (34) converges for small distances, i.e., for $z \ll a$, to the PA result. On the other hand, for large distances, i.e., for $z \gg a$, this correction should be inversely proportional to the correlation length and can be described quantitatively utilizing the LDA of the proper self-energy in Eqs. (22) and (23). In Fig. 13 we show a plot of $\Delta D^{\mathrm{E}}$ for $\mathrm{SiC}$ at the frequency $\omega=10^{14} \mathrm{~s}^{-1}$ using the roughness parameters $\delta=5 \mathrm{~nm}$ and $a=100,200$, and $500 \mathrm{~nm}$. It can be seen that the LDOS converges to the two limits for small and large distances giving a surface roughness correction on the order of some percent only for distances smaller than $100 \mathrm{~nm}$. The arrow indicates that the surface roughness correction decreases when increasing the correlation length $a$.

In Fig. 14 we show a similar plot when choosing the surface phonon frequency $\omega=\omega_{\mathrm{s}}=1.787 \times 10^{14} \mathrm{~s}^{-1}$ and plotting the modulus of $\Delta D^{\mathrm{E}}$. As before, it can be observed that $\Delta D^{\mathrm{E}}$ converges to the PA and the LDA for $z \ll a$ or $z \gg a$, respectively. Apart from that for distances $z \approx a$ the surface roughness correction to the $\operatorname{LDOS} D^{\mathrm{E}}$ becomes negative, a feature not seen for $\omega=10^{14} \mathrm{~s}^{-1}$. This behavior can be understood from the above given discussion: the dispersion re-

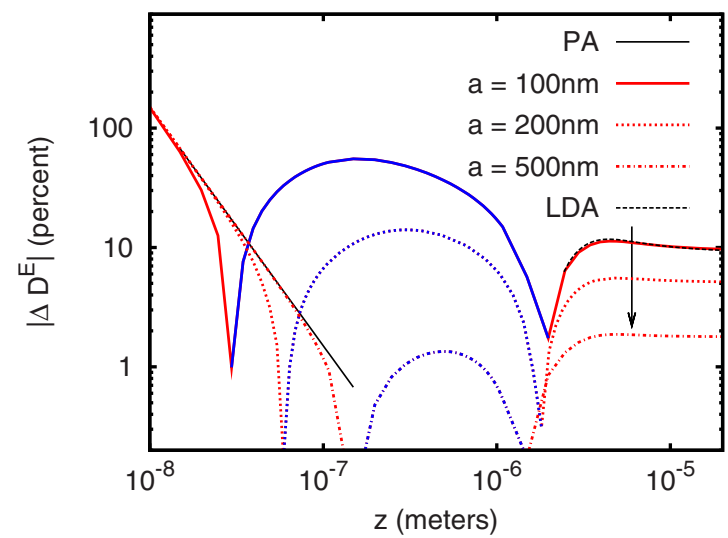

FIG. 14. (Color online) As Fig. 13 but for $\omega=1.787 \times 10^{14} \mathrm{~s}^{-1}$. The red part of the curves indicates positive values and the blue one negative values. 


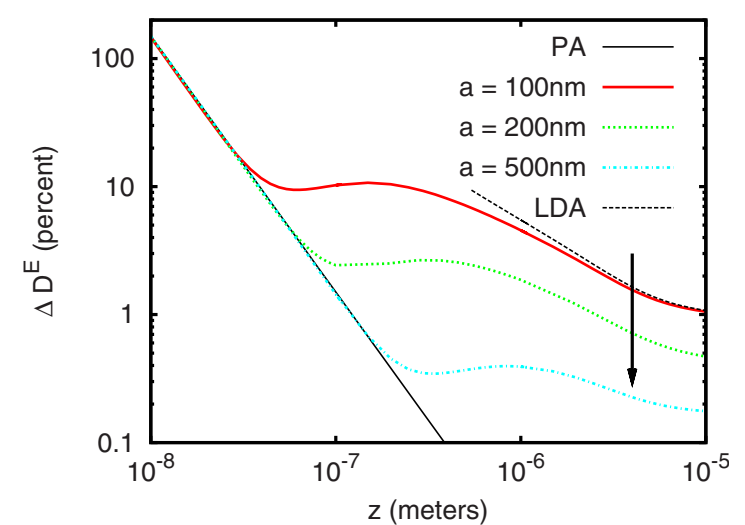

FIG. 15. (Color online) As Fig. 13 but with $\omega=1.8 \times 10^{14} \mathrm{~s}^{-1}$.

lation of the surface phonons is broadened due to roughness induced scattering yielding a negative $\Delta \operatorname{Im}\left(r_{\mathrm{p}}\right)$ for frequencies around the surface phonon frequency and for wave vectors around $a^{-1}$ because in that region the roughness induced scattering of surface phonons is strong. Since for a given distance $z$ the main contributions to $D^{\mathrm{E}}$ stem from wave vectors $\kappa \approx z^{-1}$, this roughness induced broadening becomes important for distances $z \approx a$ giving a smaller LDOS than for a flat surface so that $\Delta D^{\mathrm{E}}$ is negative. As can be seen in Fig. 14 the correction varies from about $+10 \%$ to about $-50 \%$ in the distance regime $z>100 \mathrm{~nm}$ for $a=100 \mathrm{~nm}$.

Now, as can, for example, be seen for $\Delta \operatorname{Im}\left(r_{\mathrm{p}}\right)$ in Fig. 9(b) and for the spectrum of $\Delta D^{\mathrm{E}}$ in Fig. 12 for frequencies slightly below or above the surface resonance the LDOS increases due to the roughness induced scattering of surface phonons. Hence, relatively large positive surface roughness correction can be expected for such frequencies. To illustrate this statement we also plot $\Delta D^{\mathrm{E}}$ for $\omega=1.8 \times 10^{14} \mathrm{~s}^{-1}$ in Fig. 15.

It is apparent from the above given examples that the distance dependence of the surface roughness correction to the LDOS is not only sensitive to the surface roughness parameters themselves but also to the chosen frequency. Additionally, the surface roughness correction $\Delta D^{\mathrm{E}}$ is in general nonmonotonous and especially large for frequencies around the surface phonon resonance.

\section{SUMMARY}

In this work, we studied the near-field radiative heat transfer between a nanoparticle and a rough surface utilizing direct perturbation theory up to the lowest nonvanishing order in the surface profile. Employing the material properties of $\mathrm{SiC}$ we have shown that the distance dependence of the roughness correction to the heat flux is nonmonotonous and can be qualitatively understood from the roughness correction to the LDOS.

We have derived an approximation in the small distance regime, i.e., for distances $d$ much smaller than the correlation length $a$ and have shown that it exactly coincides with the results of the proximity approximation. Hence, the roughness correction is well described by the PA for $d \ll a$. Therefrom, one can conclude that the PA might also be helpful in other geometries as used in recent experimental setups ${ }^{5-8}$ to estimate the impact of surface roughness to the near-field radiative heat transfer at small distances. Since, the numerical results give larger values for the heat transfer than predicted by the PA for most distances $d$, one can expect to get an estimate of the lower limit of the surface roughness correction when using the PA.

In the large distance limit $d \gg a$ we have derived a simple approximation for the corresponding expressions of the LDOS and the heat flux, which simplifies the numerical calculations in this limit tremendously. We have shown that in this regime the rough surface can be replaced by an equivalent surface layer of thickness $\delta^{2} / a$ for correlation lengths $a$ smaller than the skin depth making contact with the results of Ref. 31. The corrections to the heat transfer are therefore relatively small in that regime when considering roughness with $a \gg \delta$.

In the intermediate regime we could show that the LDOS and heat flux for a rough material can be smaller than that of a material with a flat surface due to the roughness induced scattering of surface phonon polaritons. Furthermore, we have shown that due to surface roughness the LDOS and therefore the heat flux has an $s$-polarized surface polariton contribution. The mechanisms behind these two unexpected results have been discussed. Finally, we want to emphasize that the results for the LDOS presented in this work have a much larger range of applicability since the LDOS, for instance, also determines the lifetime of atoms and molecules near a surface.

\section{ACKNOWLEDGMENTS}

S.-A.B. gratefully acknowledges support from the Deutsche Akademie der Naturforscher Leopoldina (Grant No. LPDS 2009-7).

\section{APPENDIX A: PERTURBATION RESULT FOR GREEN'S FUNCTION}

With the procedure in Ref. 23 and the perturbation theory in Ref. 22 it is possible to determine the Green's dyadic for each order with respect to the surface profile. The zerothand first-order expressions can be found in Ref. 23. For the second-order Green's dyadic we find for $z<z^{\prime}$,

$$
\mathbb{G}^{\mathrm{E}^{(2)}}\left(\mathbf{r}, \mathbf{r}^{\prime}\right)=\frac{1}{2} \int \frac{d^{2} \boldsymbol{\kappa}}{(2 \pi)^{2}} e^{i\left(\boldsymbol{\kappa} \cdot \mathbf{x}+\gamma_{\mathrm{r}} z\right)} \mathbb{G}^{\mathrm{E}^{(2)}}(\boldsymbol{\kappa})
$$

with

$$
\begin{aligned}
G^{\mathrm{E}^{(2)}(\boldsymbol{\kappa})=} & k_{0}^{2} \frac{1-\epsilon}{2 \gamma_{\mathrm{r}}}\left[\int \frac{d^{2} \kappa^{\prime}}{(2 \pi)^{2}} \tilde{S}^{(2)}\left(\boldsymbol{\kappa}^{\prime}-\boldsymbol{\kappa}\right)\right. \\
& \times \mathcal{T}\left(k_{\mathrm{r}}^{+}, k_{\mathrm{r}}^{+}\right) \mathbb{N}\left(k_{\mathrm{r}}^{+}, k_{\mathrm{t}}^{-\prime}\right) \mathcal{T}\left(k_{\mathrm{t}}^{-\prime}, k_{\mathrm{r}}^{-\prime}\right) f\left(\boldsymbol{\kappa}^{\prime}, z^{\prime}\right) \\
& +\int \frac{d^{2} \kappa^{\prime}}{(2 \pi)^{2}} \int \frac{d^{2} \kappa^{\prime \prime}}{(2 \pi)^{2}} \widetilde{S}^{(1)}\left(\boldsymbol{\kappa}^{\prime}-\boldsymbol{\kappa}\right) \tilde{S}^{(1)}\left(\boldsymbol{\kappa}^{\prime \prime}-\boldsymbol{\kappa}^{\prime}\right) \\
& \times 2\left(\gamma_{\mathrm{t}}^{\prime}-\gamma_{\mathrm{r}}^{\prime}\right) \mathcal{T}\left(k_{\mathrm{r}}^{+}, k_{\mathrm{r}}^{+}\right) \mathbb{L}\left(k_{\mathrm{r}}^{+}, k_{\mathrm{t}}^{-\prime}, k_{\mathrm{t}}^{-\prime \prime}\right)
\end{aligned}
$$




$$
\left.\times \mathcal{T}\left(k_{\mathrm{t}}^{-\prime \prime}, k_{\mathrm{r}}^{-\prime \prime}\right) f\left(\boldsymbol{\kappa}^{\prime \prime}, z^{\prime}\right)\right],
$$

where

$$
\begin{gathered}
f\left(\boldsymbol{\kappa}, z^{\prime}\right)=\frac{i}{2 \gamma_{\mathrm{r}}} e^{-i \boldsymbol{\kappa} \cdot \mathbf{x}^{\prime}+i \gamma_{\mathrm{r}} z^{\prime}}, \\
\widetilde{S}^{(n)}\left(\boldsymbol{\kappa}-\boldsymbol{\kappa}^{\prime}\right)=\int d^{2} x e^{i \mathbf{x} \cdot\left(\boldsymbol{\kappa}-\boldsymbol{\kappa}^{\prime}\right)}[S(\mathbf{x})]^{n}
\end{gathered}
$$

for $n=1,2$. Here all matrices $\mathrm{A}$ are defined by

$$
\mathrm{A}_{i, j}\left(k_{\mathrm{r}}^{ \pm}, k_{\mathrm{t}}^{ \pm \prime}\right)=A_{i j} \hat{\mathbf{a}}_{i}\left(k_{\mathrm{r}}^{ \pm}\right) \otimes \hat{\mathbf{a}}_{j}\left(k_{\mathrm{t}}^{ \pm \prime}\right),
$$

where $i, j=(s, p)$ and

$$
\hat{\mathbf{a}}_{\mathrm{s}}\left(k^{ \pm}\right)=\hat{\mathbf{z}} \times \hat{\boldsymbol{\kappa}} \quad \text { and } \quad \hat{\mathbf{a}}_{\mathrm{p}}\left(k^{ \pm}\right)=\hat{\mathbf{a}}_{\mathrm{s}} \times \frac{\mathbf{k}^{ \pm}}{\left|\mathbf{k}^{ \pm}\right|}
$$

are the normalized and orthogonal polarization vectors; $\hat{\mathbf{z}}$ is the unit vector in $z$ direction, $\hat{\boldsymbol{\kappa}}=\boldsymbol{\kappa} / \boldsymbol{\kappa}$ and $\mathbf{k}^{ \pm}=(\boldsymbol{\kappa}, \pm \gamma)^{t}$.

The matrix $\mathcal{T}$ is given by the diagonal matrix

$$
\mathcal{T}=\left(\begin{array}{cc}
\frac{2 \gamma_{\mathrm{r}}}{\gamma_{\mathrm{r}}+\gamma_{\mathrm{t}}} & 0 \\
0 & \frac{2 \sqrt{\epsilon} \gamma_{\mathrm{r}}}{\epsilon \gamma_{\mathrm{r}}+\gamma_{\mathrm{t}}}
\end{array}\right)=\left(\begin{array}{cc}
t_{\mathrm{s}} & 0 \\
0 & t_{\mathrm{p}}
\end{array}\right),
$$

defining the amplitude transmission coefficients $t_{\mathrm{s}}$ and $t_{\mathrm{p}}$. The components of the matrices $\mathbb{N}\left(k_{\mathrm{r}}^{+}, k_{\mathrm{t}}^{-\prime}\right)$ and $\mathrm{L}\left(k_{\mathrm{r}}^{+}, k_{\mathrm{t}}^{-\prime}, k_{\mathrm{t}}^{-\prime \prime}\right)$ are given by

$$
\begin{aligned}
& N_{\mathrm{ss}}=-\hat{\boldsymbol{\kappa}} \cdot \hat{\boldsymbol{\kappa}}^{\prime}\left(\gamma_{\mathrm{t}}+\gamma_{\mathrm{t}}^{\prime}\right), \\
& N_{\mathrm{sp}}=-\frac{\gamma_{\mathrm{t}}^{\prime}}{\sqrt{\bar{\epsilon} k_{0}}}\left(\hat{\boldsymbol{\kappa}} \times \hat{\boldsymbol{\kappa}}^{\prime}\right)_{z}\left(\gamma_{\mathrm{t}}+\gamma_{\mathrm{t}}^{\prime}\right), \\
& N_{\mathrm{ps}}=-\frac{\left(\hat{\boldsymbol{\kappa}} \times \hat{\boldsymbol{\kappa}}^{\prime}\right)_{z}}{\sqrt{\boldsymbol{\epsilon}} k_{0}}\left(\gamma_{\mathrm{r}}^{2} \boldsymbol{\epsilon}+\gamma_{\mathrm{t}} \gamma_{\mathrm{t}}^{\prime}\right), \\
& N_{\mathrm{pp}}=\frac{1}{\epsilon k_{0}^{2}}\left[-\kappa \kappa^{\prime}\left(\gamma_{\mathrm{t}}+\gamma_{\mathrm{t}}^{\prime} \boldsymbol{\epsilon}\right)+\hat{\boldsymbol{\kappa}} \cdot \hat{\boldsymbol{\kappa}}^{\prime} \gamma_{\mathrm{t}}^{\prime}\left(\gamma_{\mathrm{r}}^{2} \boldsymbol{\epsilon}+\gamma_{\mathrm{t}} \gamma_{\mathrm{t}}^{\prime}\right)\right]
\end{aligned}
$$

and

$$
\begin{aligned}
L_{\mathrm{ss}}=\left(\hat{\boldsymbol{\kappa}} \cdot \hat{\boldsymbol{\kappa}}^{\prime}\right)\left(\hat{\boldsymbol{\kappa}}^{\prime} \cdot \hat{\boldsymbol{\kappa}}^{\prime \prime}\right)-\frac{\gamma_{\mathrm{r}}^{\prime} \gamma_{\mathrm{t}}^{\prime}}{\boldsymbol{\kappa}^{\prime 2}+\gamma_{\mathrm{r}}^{\prime} \gamma_{\mathrm{t}}^{\prime}}\left(\hat{\boldsymbol{\kappa}} \times \hat{\boldsymbol{\kappa}}^{\prime}\right)_{z}\left(\hat{\boldsymbol{\kappa}}^{\prime} \times \hat{\boldsymbol{\kappa}}^{\prime \prime}\right)_{z}, \\
L_{\mathrm{sp}}=-\frac{\gamma_{\mathrm{t}}^{\prime \prime}}{\sqrt{\epsilon} k_{0}}\left(\hat{\boldsymbol{\kappa}}^{\prime} \times \hat{\boldsymbol{\kappa}}^{\prime \prime}\right)_{z}\left(\hat{\boldsymbol{\kappa}} \cdot \hat{\boldsymbol{\kappa}}^{\prime}\right) \\
-\frac{\gamma_{\mathrm{t}}^{\prime}}{\sqrt{\epsilon} k_{0}}\left(\hat{\boldsymbol{\kappa}} \times \hat{\boldsymbol{\kappa}}^{\prime}\right)_{z} \frac{\boldsymbol{\kappa}^{\prime} \boldsymbol{\kappa}^{\prime \prime}+\gamma_{\mathrm{r}}^{\prime} \gamma_{\mathrm{t}}^{\prime \prime} \hat{\boldsymbol{\kappa}}^{\prime} \cdot \hat{\boldsymbol{\kappa}}^{\prime \prime}}{\boldsymbol{\kappa}^{\prime 2}+\gamma_{\mathrm{r}}^{\prime} \gamma_{\mathrm{t}}^{\prime}},
\end{aligned}
$$

$$
\begin{aligned}
& L_{\mathrm{ps}}=-\frac{\gamma_{\mathrm{t}}}{\sqrt{\epsilon} k_{0}}\left(\hat{\boldsymbol{\kappa}} \times \hat{\boldsymbol{\kappa}}^{\prime}\right)_{z}\left(\hat{\boldsymbol{\kappa}}^{\prime} \cdot \hat{\boldsymbol{\kappa}}^{\prime \prime}\right) \\
&+\frac{\gamma_{\mathrm{t}}^{\prime}}{\sqrt{\epsilon} k_{0}}\left(\hat{\boldsymbol{\kappa}}^{\prime} \times \hat{\boldsymbol{\kappa}}^{\prime \prime}\right)_{z} \frac{\boldsymbol{\kappa} \boldsymbol{\kappa}^{\prime} \boldsymbol{\epsilon}-\gamma_{\mathrm{t}} \gamma_{\mathrm{t}}^{\prime} \hat{\boldsymbol{\kappa}} \cdot \hat{\boldsymbol{\kappa}}^{\prime}}{\boldsymbol{\kappa}^{\prime 2}+\gamma_{\mathrm{r}}^{\prime} \gamma_{\mathrm{t}}^{\prime}}, \quad(\mathrm{A} \\
& L_{\mathrm{pp}}=\frac{\gamma_{\mathrm{t}}^{\prime} \gamma_{\mathrm{t}}^{\prime \prime}}{\epsilon k_{0}^{2}}\left(\hat{\boldsymbol{\kappa}} \times \hat{\boldsymbol{\kappa}}^{\prime}\right)_{z}\left(\hat{\boldsymbol{\kappa}}^{\prime} \times \hat{\boldsymbol{\kappa}}^{\prime \prime}\right)_{z} \\
&+\frac{1}{\sqrt{\boldsymbol{\epsilon}} k_{0}^{2}} \frac{\boldsymbol{\kappa}^{\prime} \kappa^{\prime \prime}+\hat{\boldsymbol{\kappa}}^{\prime} \cdot \hat{\boldsymbol{\kappa}}^{\prime \prime} \gamma_{\mathrm{r}}^{\prime} \gamma_{\mathrm{t}}^{\prime \prime}}{\boldsymbol{\kappa}^{\prime 2}+\gamma_{\mathrm{r}}^{\prime} \gamma_{\mathrm{t}}^{\prime}}\left(\kappa \kappa^{\prime} \sqrt{\boldsymbol{\epsilon}}-\gamma_{\mathrm{t}} \gamma_{\mathrm{t}}^{\prime} \hat{\boldsymbol{\kappa}} \cdot \hat{\boldsymbol{\kappa}}^{\prime}\right) .
\end{aligned}
$$

\section{APPENDIX B: DEFINITION OF THE PROPER SELF-ENERGY}

Inserting the second-order Green's function in Eq. (A1) into Eq. (3) and averaging allows for finding the secondorder surface roughness correction to the LDOS in Eqs. (10)-(12) when defining the proper self-energy $M_{\mathrm{s} / \mathrm{p}}$ as

$$
\begin{aligned}
M_{\mathrm{s} / \mathrm{p}}= & \frac{\left(k_{0} \delta\right)^{2}(-i)(\epsilon-1)}{8 \gamma_{\mathrm{r}}^{2}\left(D_{\mathrm{s} / \mathrm{p}}^{0}\right)^{2}}\left\{N_{\mathrm{s} / \mathrm{p}}^{a}(\kappa, \kappa)+2 \int \frac{d^{2} \boldsymbol{\kappa}^{\prime}}{(2 \pi)^{2}}\right. \\
& \left.\times g\left(\left|\boldsymbol{\kappa}-\boldsymbol{\kappa}^{\prime}\right|\right) N_{\mathrm{s} / \mathrm{p}}^{b}\left(\kappa, \kappa^{\prime}, \kappa\right)\left(\gamma_{\mathrm{t}}^{\prime}-\gamma_{\mathrm{r}}^{\prime}\right)\right\}
\end{aligned}
$$

with $N_{\mathrm{s} / \mathrm{p}}^{a / b}$ given by

$$
\begin{gathered}
N_{\mathrm{s}}^{a}(\kappa, \kappa)=\left(t_{\mathrm{s}}\right)^{2} N_{\mathrm{ss}}\left(k_{\mathrm{r}}^{+}, k_{\mathrm{t}}^{-}\right), \\
N_{\mathrm{p}}^{a}(\kappa, \kappa)=\left(t_{\mathrm{p}}\right)^{2} N_{\mathrm{pp}}\left(k_{\mathrm{r}}^{+}, k_{\mathrm{t}}^{-}\right) h_{\mathrm{p}}, \\
N_{\mathrm{s}}^{b}\left(\kappa, \kappa^{\prime}, \kappa\right)=\left(t_{\mathrm{s}}\right)^{2} L_{\mathrm{ss}}\left(k_{\mathrm{r}}^{+}, k_{\mathrm{t}}^{-\prime}, k_{\mathrm{t}}^{-}\right), \\
N_{\mathrm{p}}^{b}\left(\kappa, \kappa^{\prime}, \kappa\right)=\left(t_{\mathrm{p}}\right)^{2} L_{\mathrm{pp}}\left(k_{\mathrm{r}}^{+}, k_{\mathrm{t}}^{-\prime}, k_{\mathrm{t}}^{-}\right) h_{\mathrm{p}} .
\end{gathered}
$$

Now, inserting the above defined components of the matrices $\mathbb{N}$ and $\mathbb{L}$ yields

$$
\begin{aligned}
M_{\mathrm{S}}= & -i\left(k_{0} \delta\right)^{2}(\epsilon-1) \gamma_{\mathrm{t}}+\left(k_{0} \delta\right)^{2}(\epsilon-1)^{2} k_{0}^{2} \int \frac{d^{2} \boldsymbol{\kappa}^{\prime}}{(2 \pi)^{2}} g\left(\left|\boldsymbol{\kappa}-\boldsymbol{\kappa}^{\prime}\right|\right) \\
& \times\left[D_{\mathrm{s}}^{0}\left(\boldsymbol{\kappa}^{\prime}\right)\left(\hat{\boldsymbol{\kappa}} \cdot \hat{\boldsymbol{\kappa}}^{\prime}\right)^{2}+\frac{D_{\mathrm{p}}^{0}\left(\boldsymbol{\kappa}^{\prime}\right)}{k_{0}^{2} \epsilon} \gamma_{\mathrm{r}}^{\prime} \gamma_{\mathrm{t}}^{\prime}\left(\hat{\boldsymbol{\kappa}} \times \hat{\boldsymbol{\kappa}}^{\prime}\right)^{2}\right], \quad(\mathrm{B} 6) \\
M_{\mathrm{p}}= & i\left(k_{0} \delta\right)^{2} \frac{(\epsilon-1)}{\epsilon} \gamma_{\mathrm{t}}\left(1-\frac{\kappa^{2}}{k_{0}^{2}} \frac{\epsilon+1}{\epsilon}\right) \\
& +\left(k_{0} \delta\right)^{2} \frac{(\epsilon-1)^{2}}{\epsilon^{2}} \int \frac{d^{2} \boldsymbol{\kappa}^{\prime}}{(2 \pi)^{2}} g\left(\left|\boldsymbol{\kappa}-\boldsymbol{\kappa}^{\prime}\right|\right) \\
& \times\left[-\gamma_{\mathrm{t}}^{2} D_{\mathrm{s}}^{0}\left(\kappa^{\prime}\right)\left(\hat{\boldsymbol{\kappa}} \times \hat{\boldsymbol{\kappa}}^{\prime}\right)^{2}+\frac{D_{\mathrm{p}}^{0}\left(\kappa^{\prime}\right)}{k_{0}^{2} \epsilon}\left(\kappa \kappa^{\prime}+\hat{\boldsymbol{\kappa}} \cdot \hat{\boldsymbol{\kappa}}^{\prime} \gamma_{\mathrm{r}}^{\prime} \gamma_{\mathrm{t}}\right)\right. \\
& \left.\times\left(\kappa \kappa^{\prime} \epsilon-\hat{\boldsymbol{\kappa}} \cdot \hat{\boldsymbol{\kappa}}^{\prime} \gamma_{\mathrm{t}}^{\prime} \gamma_{\mathrm{t}}\right)\right] .
\end{aligned}
$$

Finally, these expressions can be further simplified by utiliz- 
ing cylindrical coordinates and introducing the modified Bessel functions

$$
I_{n}(x)=\frac{1}{2 \pi} \int_{0}^{2 \pi} d \varphi \cos (n \varphi) e^{-x \cos (\varphi)}
$$

we get for $s$ - and $p$-polarized modes the relation

$$
M_{\mathrm{s} / \mathrm{p}}=M_{\mathrm{s} / \mathrm{p}, 0}+M_{\mathrm{s} / \mathrm{p}, 1}
$$

with

$$
\begin{aligned}
& M_{\mathrm{s}, 0}=-i\left(k_{0} \delta\right)^{2} \gamma_{\mathrm{t}}(\epsilon-1) \\
& M_{\mathrm{s}, 1}= \epsilon V \int_{0}^{\infty} d x x e^{-x^{2}}\left\{k_{0}^{2} \epsilon D_{\mathrm{s}}^{0}\left(2 x a^{-1}\right)\left[I_{0}(\kappa a x)+I_{2}(\kappa a x)\right]\right. \\
&+D_{\mathrm{p}}^{0}\left(2 x a^{-1}\right) \gamma_{\mathrm{r}}\left(2 x a^{-1}\right) \gamma_{\mathrm{t}}\left(2 x a^{-1}\right) \\
&\left.\times\left[I_{0}(\kappa a x)-I_{2}(\kappa a x)\right]\right\}
\end{aligned}
$$

and

$$
\begin{aligned}
& M_{\mathrm{p}, 0}=i\left(k_{0} \delta\right)^{2} \gamma_{\mathrm{t}} \frac{(\epsilon-1)}{\epsilon}\left(1-\frac{\kappa^{2}}{k_{0}^{2}} \frac{\epsilon+1}{\epsilon}\right), \\
M_{\mathrm{p}, 1}= & V \int_{0}^{\infty} d x x e^{-x^{2}}\left\{\frac { 2 } { \epsilon k _ { 0 } ^ { 2 } } D _ { \mathrm { p } } ^ { 0 } ( 2 x a ^ { - 1 } ) \left\{\epsilon\left(\frac{2 x \kappa}{a}\right)^{2} I_{0}(\kappa a x)\right.\right. \\
& +\gamma_{\mathrm{t}}(\kappa)\left[\gamma_{\mathrm{r}}\left(2 x a^{-1}\right) \epsilon-\gamma_{\mathrm{t}}\left(2 x a^{-1}\right)\right]\left(2 x a^{-1} \kappa\right) I_{1}(\kappa a x) \\
& \left.-\frac{1}{2}\left[I_{0}(\kappa a x)+I_{2}(\kappa a x)\right] \gamma_{\mathrm{r}}\left(2 x a^{-1}\right) \gamma_{\mathrm{t}}\left(2 x a^{-1}\right) \gamma_{\mathrm{t}}^{2}(\kappa)\right\} \\
& \left.-D_{\mathrm{s}}^{0}\left(2 x a^{-1}\right) \gamma_{\mathrm{t}}^{2}(\kappa)\left[I_{0}(\kappa a x)-I_{2}(\kappa a x)\right]\right\} .
\end{aligned}
$$

\section{APPENDIX C: APPROXIMATION OF THE PROPER SELF-ENERGY FOR $\left|k_{0} \sqrt{\epsilon} a / 2\right| \ll 1$}

Employing $\left|k_{0} \sqrt{\epsilon} a / 2\right| \ll 1$ for $\gamma_{\mathrm{r}}, \gamma_{\mathrm{t}}$, and $D_{\mathrm{s}, \mathrm{p}}^{0}$ we find for $M_{\mathrm{s}, 1}$ given in Eq. (B11)

$$
\frac{M_{\mathrm{s}, 1}}{V} \approx\left\{\epsilon^{2} k_{0}^{2} \frac{a}{4}\left[I_{0}^{0}(\kappa a)+I_{2}^{0}(\kappa a)\right]-\frac{\epsilon^{2}}{\epsilon+1} \frac{2}{a}\left[I_{0}^{2}(\kappa a)-I_{2}^{2}(\kappa a)\right]\right\},
$$

where

$$
V=\left(k_{0} \delta\right)^{2} \frac{(\epsilon-1)^{2}}{\epsilon^{2}} e^{-\kappa^{2} a^{2} / 4}
$$

and

$$
I_{n}^{m}(\xi)=\int_{0}^{\infty} d x x^{m} e^{-x^{2}} I_{n}(\xi x)
$$

For the $p$-polarized modes we get

$$
\begin{aligned}
\frac{M_{\mathrm{p}, 1}}{V} \approx & \left\{\frac { 2 } { \epsilon k _ { 0 } ^ { 2 } } \frac { \epsilon + 1 } { \epsilon } \frac { a } { 2 } \left\{(\epsilon-1) \gamma_{\mathrm{t}}(\kappa) \frac{4}{a^{2}} \kappa I_{1}^{2}(\kappa a)\right.\right. \\
& \left.+\epsilon\left(\frac{2 \kappa}{a}\right)^{2} I_{0}^{2}(\kappa a)+\frac{2}{a} \gamma_{\mathrm{t}}^{2}(\kappa)\left[I_{0}^{2}(\kappa a)+I_{2}^{2}(\kappa a)\right]\right\} \\
& \left.-\frac{a}{2} \gamma_{\mathrm{t}}^{2}(\kappa)\left[I_{0}^{0}(\kappa a)-I_{2}^{0}(\kappa a)\right]\right\} .
\end{aligned}
$$

The integrals of modified Bessel functions can be integrated analytically yielding 40

$$
\begin{gathered}
I_{n}^{0}(\xi)=\frac{\sqrt{\pi}}{2} e^{\xi^{2} / 8} I_{n / 2}\left(\frac{\xi^{2}}{8}\right), \\
I_{n}^{2}(\xi)=\frac{\sqrt{\pi}}{2} e^{\xi^{2} / 8}\left\{\left(\frac{1}{2}+\frac{\xi^{2}}{8}\right) I_{n / 2}\left(\frac{\xi^{2}}{8}\right),\right. \\
\left.+\frac{\xi^{2}}{16}\left[I_{(n-2) / 2}\left(\frac{\xi^{2}}{8}\right)+I_{(n+2) / 2}\left(\frac{\xi^{2}}{8}\right)\right]\right\}
\end{gathered}
$$

By means of these relations one can implement both limits $\kappa a \ll 1$ and $\kappa a \gg 1$ by approximating the modified Bessel function.

\section{APPENDIX D: LARGE DISTANCE LIMIT $\left(z \gg a\right.$ AND $\left.a \gg d_{\mathrm{s}}\right)$}

With a similar procedure as above but in the opposite limit $\left|k_{0} \sqrt{\epsilon} a / 2\right| \gg 1$ we find for $z \gg a$ the lowest-order terms of the self-energy

$$
\begin{gathered}
M_{\mathrm{s}, 1} \approx\left(k_{0} \delta\right)^{2} \frac{(\epsilon-1)^{2}}{\epsilon^{2}} \frac{2}{\epsilon k_{0}^{2}}\left(-i \gamma_{\mathrm{t}}^{2} k_{0} \sqrt{\epsilon}\right), \\
M_{\mathrm{p}, 1} \approx\left(k_{0} \delta\right)^{2} \frac{(\epsilon-1)^{2}}{\sqrt{\epsilon}} i k_{0} .
\end{gathered}
$$

As is apparent, in that limit the self-energy is independent from the correlation length $a$.
${ }^{1}$ K. Joulain, J.-P. Mulet, F. Marquier, R. Carminati, and J.-J. Greffet, Surf. Sci. Rep. 57, 59 (2005).

${ }^{2}$ A. I. Volokitin and B. N. J. Persson, Rev. Mod. Phys. 79, 1291 (2007).

${ }^{3}$ E. A. Vinogradov and I. A. Dorofeyev, Phys. Usp. 52, 425 (2009).

${ }^{4}$ D. Polder and M. van Hove, Phys. Rev. B 4, 3303 (1971).
${ }^{5}$ L. Hu, A. Narayanaswamy, X. Chen, and G. Chen, Appl. Phys. Lett. 92, 133106 (2008).

${ }^{6}$ E. Rousseau, A. Siria, G. Jourdan, S. Volz, F. Comin, J. Chevrier, and J.-J. Greffet, Nat. Photonics 3, 514 (2009).

${ }^{7}$ A. Narayanaswamy, S. Shen, and G. Chen, Phys. Rev. B 78, 115303 (2008).

${ }^{8}$ S. Shen, A. Narayanaswamy, and G. Chen, Nano Lett. 9, 2909 
(2009).

${ }^{9}$ R. S. DiMatteo, P. Greiff, S. L. Finberg, K. A. Young-Waithe, H. K. Choy, M. M. Masaki, and C. G. Fonstad, Appl. Phys. Lett. 79, 1894 (2001).

${ }^{10}$ A. Narayanaswamy and G. Chen, Appl. Phys. Lett. 82, 3544 (2003).

${ }^{11}$ M. Laroche, R. Carminati, and J.-J. Greffet, J. Appl. Phys. 100, 063704 (2006).

${ }^{12}$ M. Francoeur, M. P. Mengüç, and R. Vaillon, Appl. Phys. Lett. 93, 043109 (2008).

${ }^{13}$ U. F. Wischnath, J. Welker, M. Munzel, and A. Kittel, Rev. Sci. Instrum. 79, 073708 (2008).

${ }^{14}$ A. Kittel, U. F. Wischnath, J. Welker, O. Huth, F. Rüting, and S.-A. Biehs, Appl. Phys. Lett. 93, 193109 (2008).

${ }^{15} \mathrm{M}$. Nieto-Vesperinas, Scattering and Diffraction in Physical Optics (World Scientific, Singapore, 2006).

${ }^{16}$ A. A. Maradudin, Light Scattering and Nanoscale Surface Roughness (Springer Science, New York, 2007).

${ }^{17}$ J.-P. Mulet, K. Joulain, R. Carminati, and J.-J. Greffet, Appl. Phys. Lett. 78, 2931 (2001).

${ }^{18}$ F. Pincemin, A. Sentenac, and J.-J. Greffet, J. Opt. Soc. Am. A Opt. Image Sci. Vis. 11, 1117 (1994).

${ }^{19}$ L. D. Landau and E. M. Lifshitz, Statistical Physics Part 2 (Butterworth, Oxford, 2002).

${ }^{20}$ P.-O. Chapuis, M. Laroche, S. Volz, and J.-J. Greffet, Phys. Rev. B 77, 125402 (2008).

${ }^{21}$ K. Joulain, R. Carminati, J.-P. Mulet, and J.-J. Greffet, Phys. Rev. B 68, 245405 (2003).

${ }^{22}$ J.-J. Greffet, Phys. Rev. B 37, 6436 (1988).

${ }^{23}$ C. Henkel and V. Sandoghdar, Opt. Commun. 158, 250 (1998).
${ }^{24}$ A. V. Shchegrov, K. Joulain, R. Carminati, and J.-J. Greffet, Phys. Rev. Lett. 85, 1548 (2000).

${ }^{25}$ C. Genet, A. Lambrecht, P. Maia Neto, and S. Reynaud, Europhys. Lett. 62, 484 (2003).

${ }^{26}$ P. Maia Neto, A. Lambrecht, and S. Reynaud, Europhys. Lett. 69, 924 (2005).

${ }^{27}$ B. N. J. Persson, B. Lorenz, and A. I. Volokitin, Eur. Phys. J. E 31, 3 (2010).

${ }^{28}$ C. Henkel, Eur. Phys. J. D 35, 59 (2005).

${ }^{29}$ G. Nogues, C. Roux, T. Nirrengarten, A. Lupascu, A. Emmert, M. Brune, J.-M. Raimond, S. Haroche, B. Placais, and J.-J. Greffet, EPL 87, 13002 (2009).

${ }^{30}$ D. L. Brundrett, E. N. Glytsis, and T. K. Gaylord, Appl. Opt. 33, 2695 (1994).

${ }^{31}$ T. S. Rahman and A. A. Maradudin, Phys. Rev. B 21, 504 (1980).

${ }^{32}$ T. S. Rahman and A. A. Maradudin, Phys. Rev. B 21, 2137 (1980).

${ }^{33}$ G. S. Agarwal, Phys. Rev. B 15, 2371 (1977).

${ }^{34}$ A. A. Maradudin and D. L. Mills, Phys. Rev. B 11, 1392 (1975).

${ }^{35}$ A. Marvin, F. Toigo, and V. Celli, Phys. Rev. B 11, 2777 (1975).

${ }^{36}$ S. J. Elston, G. P. Bryan-Brown, and J. R. Sambles, Phys. Rev. B 44, 6393 (1991).

${ }^{37}$ F. Marquier, C. Arnold, M. Laroche, J.-J. Greffet, and Y. Chen, Opt. Express 16, 5305 (2008).

${ }^{38}$ L. A. Moraga and R. Labbé, Phys. Rev. B 41, 10221 (1990).

${ }^{39}$ G. A. Farias and A. A. Maradudin, Phys. Rev. B 28, 5675 (1983).

${ }^{40}$ I. S. Gradshteyn and I. M. Ryzhik, Table of Integrals, Series and Products (Academic Press, San Diego, 2007). 